INSTITUT NATIONAL DE RECHERCHE EN INFORMATIQUE ET EN AUTOMATIQUE

High-level Primitives for Recursive Maximum

Likelihood Estimation

Bernard C. Levy

Albert Benveniste

Ramine Nikoukhah

$\mathbf{N}^{\circ} \mathbf{2 0 8 8}$

October, 1993

PROGRAMME 5

Traitement du signal, automatique

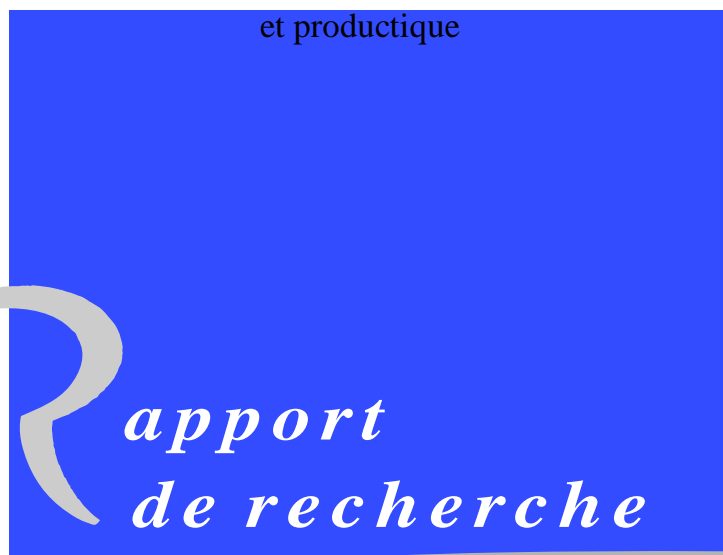





\title{
RINRIA
}

\section{High-level Primitives for Recursive Maximum Likelihood Estimation*}

\author{
Bernard C. Levy ${ }^{* *}$ \\ Albert Benveniste*** \\ Ramine Nikoukhah*** \\ Programme 5 - Traitement du signal, automatique et productique \\ Projet AS, META2 \\ Rapport de recherche $\mathrm{n}^{\circ} 2088$ - October, 1993 - 42 pages
}

\begin{abstract}
This paper proposes a high level language constituted of only a few primitives and macros for describing recursive maximum likelihood (ML) estimation algorithms. This language is applicable to estimation problems involving linear Gaussian models, or processes taking values in a finite set. The use of high level primitive allows the development of highly modular ML estimation algorithms based on only few numerical blocks. The primitives, which correspond to the combination of different measurements, the extraction of sufficient statistics, and the conversion of the status of a variable from unknown to observed, or viceversa, are first defined for linear Gaussian relations specifying mixed deterministic/stochastic information about the system variables. These primitives are used to define other macros, and are illustrated by considering the filtering and smoothing problems for linear descriptor systems. In a second stage, the primitives are extended to finite state processes, and are used to implement the Viterbi ML state sequence estimator for a hidden Markov model.
\end{abstract}

Key-words: Maximum Likelihood Estimation, Descriptor Systems, Failure Detection, Hidden Markov Models, Viterbi Algorithm.

(Résumé : tsvp)

\footnotetext{
*The research described in this paper was supported by INRIA and by the National Science Foundation under Grant MIP-9015271. It was performed while the first author was a visiting researcher at the Institut de Recherche en Informatique et Systèmes Aléatoires (IRISA) in Rennes, France.

**Department of Electrical and Computer Engineering, University of California, Davis, CA 95616.

***IRISA-INRIA, Campus Universitaire de Beaulieu, 35042 Rennes Cedex, France.

****Institut de Recherche en Informatique et Automatique (INRIA), Domaine de Voluceau, Rocquencourt, France.
}

Unité de recherche INRIA Rennes

IRISA, Campus universitaire de Beaulieu, 35042 RENNES Cedex (France)

Téléphone : (33) 99847100 - Télécopie : (33) 99847171 


\section{Primitives de haut niveau pour l'estimation récursive}

Résumé : On présente un langage de haut niveau constitué d'un petit nombre de primitives et de macros pour décrire des algorithmes récursifs d'estimation au maximum de vraisemblance. Ce langage est applicable au cas linéaire Gaussien, mais aussi au cas de variables à valeurs dans un alphabet fini.

Mots-clé : Estimation au maximum de vraisemblance, systèmes descripteurs, détection de pannes, modèles de Markov cachés, Viterbi. 


\section{Introduction}

In spite of the fact that Kalman filtering relies on a simple Gram-Schmidt orthogonalization principle, over the years, the literature devoted to Kalman filtering and smoothing [1], [2] and its square-root or fast algorithms implementations [3], [4] has become relatively complex. To deal with numerical conditioning problems, such the possible singularity of the measurement noise, or large uncertainties in the initial state variance, a number of variants of the basic filtering and smoothing algorithms have been developed. Although most of these algorithms are superficially similar, they usually require distinct implementations, which results in duplication of programming effort and a general state of confusion for the end user. There is therefore a need to develop a modular environment, where all algorithms can be constructed from a few basic modules, and where the underlying simplicity of all filtering and smoothing procedures becomes again transparent.

One could of course argue that numerical signal processing software packages, such as MATLAB, provide the desired environment for developing estimation algorithms in a modular manner. However, the functions performed by MATLAB are of a low level nature, and do not capture the statistical aspects of operations arising in recursive maximum likelihood (ML) estimation algorithms. By comparison, certain real-time signal processing languages, such as Signal [5] or its extension to random processes called Signalea [6], employ only a small number of high-level primitives, which admit simple interpretations in terms of operations on asynchronous data streams. While our goal here is more circumscribed than for SIGNAL, since we focus exclusively on estimation problems, and do not have to contend with timing issues, we shall retain the idea of employing just a few primitives to describe recursive estimation algorithms in a modular format. The objects on which the primitives operate are called observations, where an "observation" describes the hard constraints and probabilistic relationship existing between a set of measurements and unknown variables. The primitives and macros that we construct correspond in fact to simple statistical operations, such as the combination of observations, the extraction of sufficient statistics of minimal dimension, or the computation of marginal or conditional densities.

The first step towards the development of estimation primitives was taken in [7], where two operations, called reduction and extraction, were introduced to formulate linear estimation problems over graphs. The reduction operation compresses redundant observations, whereas an extraction has the effect of removing from an observation some variables which are no longer of interest. Unfortunately, the construction of the reduction and extraction operations given in [7] is nonprobabilistic and focuses only on the generation of ML estimates and error variances. As a consequence, these two operations alone cannot accomplish certain tasks, such as the generation of innovations or the computation of the likelihood function of an observation sequence. In this paper, we adopt a probabilistic point of view towards the construction of primitives. Since the statistical concepts employed to define the primitives are quite general, we do not restrict our attention to Gaussian processes. We consider all ML estimation problems that are amenable to finite computer programs, i.e. those for which conditional densities can be finitely parametrized. In addition to Gaussian models, 
these include processes taking values in a finite set, such as finite-state Markov chains [8] or Markov random fields [9].

As in [7], an important feature of the approach we employ to formulate recursive ML estimation problems is that we make no distinction between equations describing the model dynamics and measurements. In the case of dynamics, observations may involve several successive states of the process. This viewpoint is motivated by the observation that among all the equations describing a certain stochastic process, the division between a subset employed to construct the process, and another measurement subset is totally artificial. What really matters is whether, given all the available dynamics and measurements, we can estimate the variables which are of interest. This raises two issues. The first concerns the fact that any dynamic or measurement equation contains in general a mixture of deterministic and probabilistic information about the system variables. More precisely, depending on whether noise enters in an equation or not, this equation specifies either a deterministic algebraic constraint or a probability density or distribution for the system variables. This suggests that the proper probabilistic framework for modeling systems is to consider probability densities or distributions defined over submanifolds (linear subspaces in the Gaussian case) of the underlying domain. This feature appears already in Signalea[6], where stochastic processes are constructed by combining deterministic relations with a random variable generator. The fact that we process all dynamics and measurements progressively, instead of constructing the process a priori, engenders a second problem. Specifically, it is possible that certain variables which are estimable from the complete observation set, may remain unknown on the basis of the current partial observation set. Thus, we are confronted with a situation where at any given time we have mixed information about the system variables: some are known exactly, others are unknown, and the remainder admits a probability distribution.

The paper is organized as follows. We consider first the case of linear Gaussian models. In Section 2 we show that we can associate to a linear Gaussian observation a pair formed by a constraint space for the system parameters and measurements, and a Gaussian probability density defined over this space. A criterion is presented to determine whether a variable is estimable from the specification of such a pair, and the maximum likelihood and leastsquares estimates are obtained by solving constrained optimization problems. The highlevel ML estimation primitives are introduced in Section 3. The first primitive performs the combination of two observations. This involves imposing to the combined observation the deterministic constraints associated to each of its components. Another difficulty arises when the observations we combine contain common parameters or measurement vectors. The equality of these parameters and measurements must be taken into account in the combination step. The second and third primitive are formed by the reduction operation and its complement, the coreduction. From a statistical point of view, the reduction of an observation generates a minimal sufficient statistic while preserving the constraint space of the parameter vector. The coreduction extracts from an observation the information concerning only its measurement vector. It yields the likelihood function of the measurement vector, as well as the deterministic constraints, often called parity checks, that must be applied to the measurement to determine whether it is compatible with the observation 
model. The last primitive, called mutation, is needed to define conditional observations, and consists of switching the status of a parameter or measurement from unknown to known, or vice-versa. In our framework, the extraction operation of [7] is only a macro, which can be implemented easily in terms of the four primitives. Efficient numerical implementations of the primitives are also provided, so that to each primitive corresponds both a statistical concept and a numerical module.

The primitives and macros are used to obtain a general recursion principle for the computation of ML estimates, and the evaluation of the likelihood function and parity checks of a stream of observations. This principle is illustrated in Section 4 by considering the filtering and smoothing of linear Gaussian descriptor systems, as well as a simple failure detection problem. High level programs are provided for the Kalman filter, and the two-filter and Rauch-Tung-Striebel smoothers. These programs are so compact it is hard to believe they implement a complete Kalman filter or smoother. However, by keeping track of the step by step implementation of each primitive, it is easy to relate them to standard Kalman filter or smoother implementations.

The case of a parameter process taking values in a finite set is considered in Section 5. Depending on whether the measurements are continuous or discrete, an observation is modeled as a probabilility density or distribution defined over a constraint manifold, corresponding to the deterministic constraints imposed on the parameters and measurement. Because of the discrete nature of the parameter process, only ML estimates are of interest. Also, since the primitives correspond to general statistical operations, it is not difficult to adapt them to the given model. The only difference is that depending on whether the primitives act on Gaussian or finite-valued variables, they rely on different numerical algorithms. The applicability of the primitives is illustrated by considering the maximum likelihood state sequence estimation problem for a hidden Markov model. By realizing that this problem has the same graph dependency structure between observations and parameters as the linear descriptor estimation problem of Section 4 (see [7] and Section 5 for a discussion of the XO-graph representation of an estimation problem), we demonstrate, somewhat surprisingly, that the Viterbi algorithm admits the same high-level program as the double-sweep

Rauch-Tung-Striebel smoother. Thus, although the two algorithms look very different, the use of primitives sensitive to data type allows us to show they are identical, which makes precise an analogy observed in [10].

\section{Estimation over Constrained Spaces}

\section{A Observation Model}

Consider a linear observation

$$
\text { o : } E y=A x+B u
$$

where $u \sim \mathcal{N}\left(0, I_{m}\right)$ is a zero-mean Gaussian vector of $\mathbf{R}^{m}$, and the measurement $y \in \mathbf{R}^{p}$ and parameter vector $x \in \mathbf{R}^{n}$ are respectively known (we shall say observed) and unknown.

$\mathrm{RR} \mathrm{n}^{\circ} 2088$ 
The observation $o$ has dimension $q$, where $q$ denotes the dimension of the vector $E y$, so that the matrices $E, A$ and $B$ have sizes $q \times p, q \times n$ and $q \times m$, respectively. In (2.1), the covariance $B B^{T}$ of the noise $B u$ need not be invertible, but without loss of generality, it can be assumed that $B$ is one to one, i.e., $B$ has full column rank.

The observation model (2.1) is nonstandard, since it is customary to set $E=I$, with $q=p$. However, the greater generality afforded by the model (2.1) will enable us to combine observations $o_{1}$ and $o_{2}$ whose measurement vectors $y_{1}$ and $y_{2}$ contain common components. This model allows also the existence of algebraic constraints between the entries of $y$. In this context, because no constraint is imposed on the rows of $E$, it is worth noting that the distribution $u \sim \mathcal{N}\left(0, I_{m}\right)$ is an a-priori distribution for the noise, before the specification of equation (2.1). After the observation (2.1) becomes known, some components of $u$ may be known exactly. The following lemma describes more precisely the information provided by equation (2.1).

\section{Lemma 2.1 (canonical decomposition)}

1) Given a linear observation o of the form (2.1), there exists a transformation $(S, V)$ with $S$ invertible and $V$ orthonormal such that

$$
S A=\left[\begin{array}{c}
M_{1} \\
M_{2} \\
0 \\
0
\end{array}\right] \quad S B V^{T}=\left[\begin{array}{cc}
I & 0 \\
0 & 0 \\
0 & I \\
0 & 0
\end{array}\right]
$$

with $M=\left[\begin{array}{l}M_{1} \\ M_{2}\end{array}\right]$ onto, so that if

$$
S E=F=\left[\begin{array}{l}
F_{1} \\
F_{2} \\
F_{3} \\
F_{4}
\end{array}\right] \quad V u=\left[\begin{array}{l}
u_{1} \\
u_{3}
\end{array}\right],
$$

the observation o takes the form

$$
\left[\begin{array}{l}
F_{1} \\
F_{2} \\
F_{3} \\
F_{4}
\end{array}\right] y=\left[\begin{array}{c}
M_{1} \\
M_{2} \\
0 \\
0
\end{array}\right] x+\left[\begin{array}{c}
u_{1} \\
0 \\
u_{3} \\
0
\end{array}\right],
$$

where $u_{1} \sim \mathcal{N}(0, I)$ and $u_{3} \sim \mathcal{N}(0, I)$ are independent.

2) The canonical decomposition (2.2)-(2.4) provides the following information about $x$ and $y$. First, $\left[y^{T} x^{T}\right]^{T}$ belongs to the subspace $\mathcal{C}$ of $\mathbf{R}^{p+n}$ defined by the two constraints

$$
\begin{aligned}
& F_{2} y=M_{2} x \\
& F_{4} y=0 .
\end{aligned}
$$


Over $\mathcal{C}$, the observation o specifies the Gaussian density

$$
\mathbf{p}(y, x)=k \exp -\frac{1}{2}\left(\left\|\left(F_{1} y-M_{1} x\right)\right\|^{2}+\left\|F_{3} y\right\|^{2}\right)
$$

where $k$ is a constant which can be selected equal to 1 , since we do not require that $\mathbf{p}$ should be normalized ${ }^{1}$. Thus, the specification of an observation of the form (2.1) is equivalent to the selection of a Gaussian density and constraint space pair $(\mathbf{p}(y, x)$, C).

Proof: We only need to prove 1), since 2) is a direct consequence of 1). By performing a QR factorization of $A$, we can find an orthonormal matrix

$$
T=\left[\begin{array}{l}
K \\
L
\end{array}\right]
$$

such that

$$
T A=\left[\begin{array}{c}
A_{r} \\
0
\end{array}\right]
$$

where $A_{r}$ has full row rank. Denoting

$$
T E=\left[\frac{E_{r}}{E}\right]
$$

and $\bar{B}=L B$, we see that

$$
\bar{E} y=\bar{B} u \text {. }
$$

Let

$$
\bar{B}=U\left[\begin{array}{cc}
0 & \Sigma \\
0 & 0
\end{array}\right] V
$$

with $U$ and $V$ orthonormal, and $\Sigma$ diagonal positive definite, be a singular value decomposition of $\bar{B}$. Note that the block $\Sigma$ has been placed in position $(1,2)$ in order to ensure consistency with the choice (2.3) of input transformation. Then, the transformation

$$
\left[\begin{array}{l}
F_{3} \\
F_{4}
\end{array}\right]=\left[\begin{array}{cc}
\Sigma^{-1} & 0 \\
0 & I
\end{array}\right] U^{T} \bar{E} \quad\left[\begin{array}{l}
u_{1} \\
u_{3}
\end{array}\right]=V u
$$

yields the last two block rows of (2.4), where, because $V$ is orthonormal, the covariance of $V u$ is identity. This implies $u_{1}$ and $u_{3}$ are independent with unit variance. Writing

$$
K B V^{T}=\left[\begin{array}{ll}
B_{r} & C_{r}
\end{array}\right],
$$

\footnotetext{
${ }^{1}$ In other words, all densities which differ only by a multiplicative constant will be viewed as equivalent. This enables us to perform arbitrary linear transformations on the vectors $x$ and $y$ without keeping track of the Jacobians of the transformations.
}

$\mathrm{RR} \mathrm{n}^{\circ} 2088$ 
and taking into account the third block row of (2.4), we also obtain

$$
F_{r} y=A_{r} x+B_{r} u_{1}
$$

with

$$
F_{r} \triangleq E_{r}-C_{r} F_{3} .
$$

The assumption that $B$ has full column rank implies $B_{r}$ has full column rank. Consequently, by performing a QR decomposition of $B_{r}$, we can find an invertible matrix $Z$ such that

$$
Z B_{r}=\left[\begin{array}{l}
I \\
0
\end{array}\right] \text {. }
$$

Multiplying (2.13a) on the left by $Z$, and denoting

$$
\left[\begin{array}{l}
F_{1} \\
F_{2}
\end{array}\right]=Z F_{r} \quad M=\left[\begin{array}{l}
M_{1} \\
M_{2}
\end{array}\right]=Z A_{r},
$$

then gives the first two block rows of (2.4), where the matrix $M$ has full row rank since $A_{r}$ has full row rank and $Z$ is invertible.

\section{Remarks:}

1) An interesting feature of the above decomposition is that it relies exclusively on numerically stable operations, such as QR or singular value decompositions. In some sense, it can be viewed as just a formalization of procedures which occur repeatedly, but under different disguises, in the implementation of square-root Kalman filters [3].

2) Given an observation $o$ of the form (2.1), the generation of the last two equations of (2.4) requires only the first step of the procedure employed to construct (2.4). Specifically, as indicated by $(2.7 \mathrm{a})-(2.7 \mathrm{~b})$, if $L$ is a matrix whose rows form a basis of the left null space of $A$, so that $L A=0$, the last two equations of (2.4) are given by

$$
L E y=L B u \text {. }
$$

Thus, the full decomposition procedure of Lemma 2.1 needs to be applied only if we seek to compute the whole canonical form (2.4).

An interesting feature of the constraints $(2.5 \mathrm{a})$ and $(2.5 \mathrm{~b})$ defining $\mathcal{C}$ is that the second involves $y$ only. The space

$$
\mathcal{S}=\left\{y: F_{4} y=0\right\}
$$

identifies the observations which are compatible with the model (2.1), so that the test $F_{4} y=$ 0 is actually the parity check that must be applied to an observation $y$ to determine whether it can arise from the model (2.1). In this context, it is usually desirable to compress the row space of $F_{4}$, i.e to find an orthonormal matrix $W$ such that

$$
W F_{4}=\left[\begin{array}{c}
F_{4 r} \\
0
\end{array}\right]
$$


where $F_{4 r}$ has full row rank. Then, the parity check test required to determine whether $y$ belongs to $\mathcal{S}$ reduces to

$$
F_{4 r} y=0,
$$

which involves only independent tests. Furthermore, if

$$
\mathcal{X}(y)=\left\{x: M_{2} x=F_{2} y \text { for } y \in \mathcal{S}\right\},
$$

the constraint space $\mathcal{C}$ can be expressed as

$$
\mathcal{C}=\bigcup_{y \in \mathcal{S}}\left[\begin{array}{c}
y \\
\mathcal{X}(y)
\end{array}\right]
$$

i.e. given a valid observation $y \in \mathcal{S}$, the vector $x$ is constrained to belong to the affine space $\mathcal{X}(y)$. This space describes the deterministic constraints to which $x$ is subjected once $y$ has been obtained and its validity established.

The affine space $\mathcal{X}(y)$ can be parametrized as follows. Let $N_{2}$ be a matrix whose columns form a basis of the right null space of $M_{2}$. Then, observing that

$$
x_{p}(y)=M_{2}^{T}\left(M_{2} M_{2}^{T}\right)^{-1} F_{2} y
$$

is a particular solution of the equation

$$
M_{2} x=F_{2} y,
$$

the vectors of $\mathcal{X}(y)$ admit the linear parametrization

$$
x(\xi, y)=N_{2} \xi+x_{p}(y)
$$

where $\xi$ is an arbitrary vector.

Thus, once the validity of the observation $y$ has been established, the information available about $x$ presents the following features:

(i) The density $\mathbf{p}(y, x)$ is concentrated on the affine space $\mathcal{X}(y)$, so that some components of $x$ are known perfectly.

(ii) Some other components are completely unknown, which is reflected by the fact that the density $\mathbf{p}$ is uniformly distributed with respect to these variables.

(iii) The remaining components of $x$ admit a Gaussian distribution.

We are therefore confronted with a situation where we have mixed information about the various components of $x$. Obviously, given a complete set of observations, we would like to find ourselves in a situation where $x$ does not include any unknown component. This property can be described as follows.

$\mathrm{RR} \mathrm{n}^{\circ} 2088$ 
Definition 2.1 A vector $x$ is said to be estimable if, given a linear parametrization $x(\xi, y)$ of $\mathcal{X}(y)$, the $\log$-density $-\ln \mathbf{p}(y, x(\xi, y))$ is a nonsingular quadratic form of the vector $\xi$. Then, $\mathbf{p}(y, x)$ admits a unique maximum over $\mathcal{X}(y)$, and the integral

$$
\int g(x(\xi, y)) \mathbf{p}(y, x(\xi, y)) d \xi
$$

exists for all functions $g(x)$ which grow at most polynomially as $\|x\| \rightarrow \infty$, so that all moments of the density $\mathbf{p}(y, x)$ exist over $\mathcal{X}(y)$.

Setting $g=1$ in (2.24), we see that when $x$ is estimable, the marginal density

$$
\mathbf{p}(y)=\int \mathbf{p}(y, x(\xi, y)) d \xi
$$

can be defined over the space $\mathcal{S}$ of valid observations.

Lemma 2.2 The vector $x$ is estimable if and only if in the observation equation (2.1), $A$ is one-to-one, or equivalently, iff in the canonical decomposition (2.2)-(2.4), the matrix

$$
M=\left[\begin{array}{l}
M_{1} \\
M_{2}
\end{array}\right]
$$

is invertible.

Proof: Substituting the parametrization $(2.23)$ of $\mathcal{X}(y)$ inside the density $\mathbf{p}(y, x)$ in $(2.6)$ yields

$$
-\ln \mathbf{p}(y, x(\xi, y))=\frac{1}{2}\left(\left\|F_{1} y-M_{1} x_{p}(y)-M_{1} N_{2} \xi\right\|^{2}+\left\|F_{3} y\right\|^{2}\right),
$$

which shows that $-\ln \mathbf{p}(y, x(\xi, y))$ is a nonsingular quadratic form of $\xi$ if and only if $M_{1} N_{2}$ is one to one, i.e. iff no vector of the null space of $M_{2}$ belongs also to the null space of $M_{1}$. This means that $M$ must be invertible (note that by construction, $M$ is onto). Since $M$ is related to $A$ through the transformation (2.2), this is equivalent to requiring that $A$ should be one to one.

The expression (2.25) defines the marginal density $\mathbf{p}(y)$ only when $x$ is estimable. However, given an observation of the form (2.1), we can always obtain an estimable parametrization by factoring $A$ as

$$
A=N M
$$

with $N$ one-to-one and $M$ onto. Then, with $z=M x$, the observation can be rewritten as

$$
E y=N z+B u,
$$


where $z$ is estimable. Thus, we can always find a parametrization which allows the evaluation of the marginal density $\mathbf{p}(y)$ through (2.25). In particular, in the coordinate system (2.2)(2.4), we can select as estimable parameter vector

$$
z=\left[\begin{array}{l}
z_{1} \\
z_{2}
\end{array}\right]=\left[\begin{array}{l}
M_{1} \\
M_{2}
\end{array}\right] x .
$$

Integrating the density

$$
\mathbf{p}(y, z)=\exp -\frac{1}{2}\left(\left\|F_{1} y-z_{1}\right\|^{2}+\left\|F_{3} y\right\|^{2}\right)
$$

over the affine space $\mathcal{Z}(y)=\left\{z: z_{2}=F_{2} y\right\}$ then gives

$$
\mathbf{p}(y)=\exp -\frac{1}{2}\left\|F_{3} y\right\|^{2}
$$

which is defined over $\mathcal{S}$.

A pair $(\mathbf{p}(y, x), \mathcal{C})$ can therefore be decomposed into two pairs:

(i) $(\mathbf{p}(y), \mathcal{S})$ which specifies the marginal density and constraint space for the measurement $y$. The density $\mathbf{p}($.$) will be called the likelihood function of the observation o$, and will be denoted as $L(o)$. Also, unless the context is clear, we shall use $\mathcal{S}(o)$ to denote the constraint space associated to $o$, so that $\mathcal{S}^{\perp}(o)$ will be the space of parity checks that must be applied to $y$ to determine its validity.

(ii) The conditional density and constraint space pair $(\mathbf{p}(x \mid y)=\mathbf{p}(y, x) / \mathbf{p}(y), \mathcal{X}(y))$, where in the coordinate system (2.2)-(2.4), we have

$$
\mathbf{p}(x \mid y)=\exp -\frac{1}{2}\left\|F_{1} y-M_{1} x\right\|^{2} .
$$

Thus, the major difference with observation models with nonsingular noise is that we must keep track of constraint spaces along with the usual densities or conditional densities.

\section{B ML and LS Estimation}

The probabilistic set-up that we employ has the effect of blurring the usual distinction between Bayesian and ML estimation. On one hand, we model all prior information as observations. For example, to describe the measurement equation

$$
y=H x+J w
$$

with $x \sim \mathcal{N}(0, P)$ independent of $w \sim \mathcal{N}\left(0, I_{m}\right)$, we replace it by the equivalent model

$$
\left[\begin{array}{l}
0 \\
I
\end{array}\right] y=\left[\begin{array}{c}
I \\
H
\end{array}\right] x+\left[\begin{array}{cc}
G & 0 \\
0 & J
\end{array}\right]\left[\begin{array}{c}
v \\
w
\end{array}\right]
$$

$\operatorname{RR} \mathrm{n}^{\circ} 2088$ 
with $G G^{T}=P$ and the noise $v \sim \mathcal{N}\left(0, I_{r}\right)$ independent of $w$, where $r$ denotes the rank of $P$. The only difference betwen (2.34) and (2.35) is that the prior information about $x$ is modeled as an extra measurement. This gives the impression that we have adopted a pure ML point of view. Yet, unlike the usual ML philosophy, where $\mathbf{p}(y, x)$ is considered as a probability density for $y$ with $x$ unknown and fixed, $\mathbf{p}(y, x)$ is viewed here as a joint density for both $y$ and $x$ over the constraint space $\mathcal{C}$.

Then, the problem of estimating an estimable vector $x$ from an observation $o$ of the form (2.1) can be formulated as follows.

Definition 2.2 Assume that $x$ is estimable. Then the $M L$ estimate $\hat{x}_{M L}(y)$ of $x$ given $y \in \mathcal{S}$ is the argument of the maximum of $\mathbf{p}(x \mid y)$ (or equivalently, $\mathbf{p}(y, x)$ ) over $\mathcal{X}(y)$. The leastsquares $(L S)$ estimate $\hat{x}_{L S}(y)$ is the function $\hat{x}(y) \in \mathcal{X}(y)$ which minimizes

$$
E\left[\|x-\hat{x}(y)\|^{2} \mid y\right] \triangleq \int\|x(\xi, y)-\hat{x}(y)\|^{2} \mathbf{p}(x(\xi, y) \mid y) d \xi,
$$

where $x(\xi, y)$ is a linear parametrization of $\mathcal{X}(y)$.

It is well known, at least in the case of observations with nonsingular noise, that for linear Gaussian models, the ML and LS estimates are the same. This result remains true in the presence of deterministic information.

Theorem 2.1 If $x$ is estimable, its $M L$ and LS estimates are identical, i.e.,

$$
\hat{x}_{M L}(y)=\hat{x}_{L S}(y)
$$

and

$$
\mathbf{p}(y)=\mathbf{p}\left(y, \hat{x}_{M L}(y)\right)
$$

for $y \in \mathcal{S}$, where the equality in (2.376) holds modulo multiplication by a constant independent of $y$. Thus the likelihood function of o can be obtained either by computing the marginal density $\mathbf{p}(y)$ or by substituting the $M L$ estimate $\hat{x}_{M L}(y)$ inside the joint density $\mathbf{p}(y, x)$.

Proof: We need only to modify slightly the usual derivation for Gaussian densities over unconstrained spaces. Without loss of generality, it can be assumed $o$ has been brought to the canonical form (2.2)-(2.4), where since $x$ is estimable, $M$ is invertible. Denoting

$$
M^{-1}=Q=\left[\begin{array}{ll}
Q_{1} & Q_{2}
\end{array}\right],
$$

the space $\mathcal{X}(y)$ can be parametrized as

$$
x(\xi, y)=Q_{1} \xi+Q_{2} F_{2} y,
$$

which yields

$$
\mathbf{p}(x(\xi, y) \mid y)=\exp -\frac{1}{2}\left\|F_{1} y-\xi\right\|^{2} .
$$


Its maximum is reached for $\hat{\xi}_{M L}=F_{1} y$, so that

$$
\hat{x}_{M L}(y)=Q\left[\begin{array}{l}
F_{1} \\
F_{2}
\end{array}\right] y .
$$

Also, since $\mathbf{p}\left(\hat{x}_{M L}(y) \mid y\right)=1,(2.37 \mathrm{~b})$ holds.

Similarly, by employing the parametrization (2.39), we find

$$
\begin{aligned}
& \int\|x(\xi, y)-\hat{x}(y)\|^{2} \mathbf{p}(x(\xi, y)) d \xi \\
& \left.=\int\left\|Q\left[\begin{array}{c}
\xi \\
F_{2} y
\end{array}\right]-\hat{x}(y)\right\|^{2} \exp -\left\|F_{1} y-\xi\right\|^{2} / 2,\right)
\end{aligned}
$$

which is minimized by

$$
\hat{x}_{L S}(y)=Q\left[\begin{array}{l}
F_{1} \\
F_{2}
\end{array}\right] y,
$$

so that the ML and LS estimates coincide.

\section{Primitives and Macros}

We have now all the tools necessary to develop a probabilistic interpretation for the ML estimation primitives introduced in [7]. However, unlike [7], where the attention was focused exclusively on ML estimates and their associated error variances, we shall examine the effect of the primitives on all the subcomponents of an observation.

\section{A Combinator}

The first primitive we consider is the combination $o_{1} \wedge o_{2}$ of two independent observations $o_{1}$ and $o_{2}$, where the symbol " $\wedge$ " should be interpreted as the logical "and" connector.

Definition 3.1 Consider two observations

$$
\begin{array}{ll}
o_{1}: & {\left[\begin{array}{ll}
E_{11} & E_{1 c}
\end{array}\right]\left[\begin{array}{l}
y_{1} \\
y_{c}
\end{array}\right]=\left[\begin{array}{ll}
A_{11} & A_{1 c}
\end{array}\right]\left[\begin{array}{l}
x_{1} \\
x_{c}
\end{array}\right]+B_{1} u_{1}} \\
o_{2}: & {\left[\begin{array}{ll}
E_{2 c} & E_{22}
\end{array}\right]\left[\begin{array}{l}
y_{c} \\
y_{2}
\end{array}\right]=\left[\begin{array}{ll}
A_{2 c} & A_{22}
\end{array}\right]\left[\begin{array}{l}
x_{c} \\
x_{2}
\end{array}\right]+B_{2} u_{2},}
\end{array}
$$

where the noises $u_{1}$ and $u_{2}$ are independent with $u_{i} \sim \mathcal{N}(0, I)$ for $i=1$, 2, and such that $y_{c}$ and $x_{c}$ denote respectively the measurement and parameter vector components which are common to both observations. The combined observation is given by

$$
o_{1} \wedge o_{2}: \quad\left[\begin{array}{ccc}
E_{11} & E_{1 c} & 0 \\
0 & E_{2 c} & E_{22}
\end{array}\right]\left[\begin{array}{l}
y_{1} \\
y_{c} \\
y_{2}
\end{array}\right]
$$

$\mathrm{RR} \mathrm{n}^{\circ} 2088$ 


$$
=\left[\begin{array}{ccc}
A_{11} & A_{1 c} & 0 \\
0 & A_{2 c} & A_{22}
\end{array}\right]\left[\begin{array}{l}
x_{1} \\
x_{c} \\
x_{2}
\end{array}\right]+\left[\begin{array}{cc}
B_{1} & 0 \\
0 & B_{2}
\end{array}\right]\left[\begin{array}{l}
u_{1} \\
u_{2}
\end{array}\right] .
$$

The combinator $\wedge$ is clearly commutative and associative. If

$$
\begin{aligned}
y=\left[\begin{array}{l}
y_{1} \\
y_{c} \\
y_{2}
\end{array}\right] & x=\left[\begin{array}{l}
x_{1} \\
x_{c} \\
x_{2}
\end{array}\right] \\
\eta_{1}=\left[\begin{array}{l}
y_{1} \\
y_{c}
\end{array}\right] & \eta_{2}=\left[\begin{array}{l}
y_{c} \\
y_{2}
\end{array}\right] \\
\chi_{1}=\left[\begin{array}{l}
x_{1} \\
x_{c}
\end{array}\right] & \chi_{2}=\left[\begin{array}{l}
x_{c} \\
x_{2}
\end{array}\right],
\end{aligned}
$$

the constraint space $\mathcal{C}$ for $\left[y^{T} x^{T}\right]^{T}$ is obtained by taking the conjunction of the constraints defining $\mathcal{C}_{1}$ and $\mathcal{C}_{2}$, which concern respectively the vectors $\left[\begin{array}{ll}\eta_{1}^{T} & \chi_{1}^{T}\end{array}\right]^{T}$ and $\left[\begin{array}{ll}\eta_{2}^{T} & \chi_{2}^{T}\end{array}\right]^{T}$. Over $\mathcal{C}$, the density of $o_{1} \wedge o_{2}$ is given by

$$
\mathbf{p}(y, x)=\mathbf{p}_{1}\left(\eta_{1}, \chi_{1}\right) \mathbf{p}_{2}\left(\eta_{2}, \chi_{2}\right),
$$

where $\mathbf{p}_{i}\left(\eta_{i}, \chi_{i}\right)$ is the density over $\mathcal{C}_{i}$ associated to the observation $o_{i}$, with $i=1,2$.

\section{B Reduction and Coreduction}

Next, consider an observation $o$ of the form (2.1). It may contain information which is either redundant or irrelevant for the purpose of estimating $x$. This manifests itself by the existence of a sufficient statistic of smaller dimension than Ey. However, for our purposes we need a concept stronger than that of a sufficient statistic, which concerns only the compression of an observation vector. In addition, we need to ensure that the constraint space $\mathcal{X}(y)$ is not affected by the replacement of $E y$ by a sufficient statistic. This leads us to introduce the concept of sufficient observation, which is directly derived from the Neyman-Fisher factorization criterion [11], p. 39 for the sufficiency of a statistic.

Definition 3.2 Given an observation o given by (2.1), we say that

$$
o_{s}: \quad K E y=K A x+K B u
$$

is a sufficient observation for o if it has the same affine constraint space:

$$
\mathcal{X}_{s}(y)=\mathcal{X}(y)
$$

and if, over this space,

$$
\mathbf{p}_{s}(x \mid y)=\mathbf{p}(x \mid y),
$$

where $\mathbf{p}_{s}(x \mid y)$ and $\mathbf{p}(x \mid y)$ denote respectively the conditional densities (cf. (2.33)) corresponding to $o_{s}$ and $o$. 
If $\mathbf{p}(y, x)$ and $\mathbf{p}_{s}(y, x)$ are the densities of $o$ and $o_{s}$, it is easy to verify that the condition (3.7) is equivalent to requiring

$$
\mathbf{p}(y, x)=\mathbf{p}_{s}(y, x) \mathbf{q}(y)
$$

where $\mathbf{q}(y)$ is independent of $x$, which except for the fact it must hold over the affine space (3.6), takes the usual form of the Neyman-Fisher factorization criterion. A simple situation where a sufficient observation can be identified by inspection is as follows.

Lemma 3.1 Consider two observations

$$
\begin{array}{ll}
o_{0}: & E_{0} y=A_{0} x+B_{0} u_{0} \\
o_{1}: & E_{1} y=B_{1} u_{1},
\end{array}
$$

where $u_{0}$ and $u_{1}$ are two independent zero-mean Gaussian vectors with unit variance. Then, $o_{0}$ is a sufficient observation for $o=o_{0} \wedge o_{1}$.

Proof: If $\mathbf{p}_{0}(y, x)$ and $\mathbf{p}_{1}(y)$ denote respectively the the densities of $o_{0}$ and $o_{1}$, the density of the joint observation $o$ is given by

$$
\mathbf{p}(y, x)=\mathbf{p}_{0}(y, x) \mathbf{p}_{1}(y),
$$

which is in the form (3.8). Also, since $x$ does not appear in $o_{1}$, and the observation noises are independent,

$$
\mathcal{X}(y)=\mathcal{X}_{0}(y)
$$

Since all sufficient observations corresponding to a given observation need not have the same dimension, it is of interest to characterize those with the smallest size. The concept of minimal sufficient statistic [11], p. 41 can be extended to observations in the following manner.

Definition 3.3 Consider an observation o of dimension q, i.e. such Ey has dimension $q$. It is minimal if it does not admit a sufficient observation os of lower dimension.

Lemma 3.2 An observation o is minimal if and only if the matrix $A$ in (2.1) has full row rank, or equivalently, its likelihood function and parity space satisfy

$$
\mathbf{p}(y)=1 \quad \mathcal{S}^{\perp}(o)=\{0\},
$$

i.e. the observation vector is unconstrained and uniformly distributed.

Proof: Without loss of generality it can be assumed that $o$ is in the form (2.2)-(2.4), in which case

$$
A=\left[\begin{array}{c}
M_{1} \\
M_{2} \\
0 \\
0
\end{array}\right] .
$$

$\mathrm{RR} \mathbf{n}^{\circ} 2088$ 
From this representation, we can conclude $A$ has full row rank iff its canonical decomposition does not include $F_{3} y$ and $F_{4} y$ components. According to the representations (2.32) and (2.17) for $\mathbf{p}(y)$ and $\mathcal{S}(o)$, this is equivalent to (3.12).

Conversely, suppose that $A$ has full row rank, and that

$$
o_{s}: \quad K E y=K A x+K B u
$$

is a sufficient statistic for $o$. Without loss of generality, we can assume that both $o_{s}$ and $o$ are in canonical form (this requires employing different representations of the noise vector $u$ in $o$ and $o_{s}$ ), in which case

$$
\begin{array}{cc}
K E=\left[\begin{array}{c}
F_{s 1} \\
F_{s 2} \\
F_{s 3} \\
F_{s 4}
\end{array}\right] & K A=\left[\begin{array}{c}
M_{s 1} \\
M_{s 2} \\
0 \\
0
\end{array}\right] \\
E=\left[\begin{array}{c}
F_{1} \\
F_{2}
\end{array}\right] & A=\left[\begin{array}{c}
M_{1} \\
M_{2}
\end{array}\right],
\end{array}
$$

where since $A$ has full row rank, the canonical decomposition of $o$ does not include $F_{3} y$ or $F_{4} y$ components. Because $o_{s}$ is a sufficient observation for $o$, it satisfies the conditions (3.6) and (3.7). Observing that since $A$ has full row rank, the observations $y$ are unconstrained, the condition (3.6) implies that the right null spaces of

$$
\left[\begin{array}{ll}
F_{s 2} & -M_{s 2}
\end{array}\right] \text { and }\left[\begin{array}{ll}
F_{2} & -M_{2}
\end{array}\right]
$$

are identical, so that there exists an invertible matrix $U_{2}$ such that

$$
U_{2}\left[\begin{array}{ll}
F_{s 2} & -M_{s 2}
\end{array}\right]=\left[\begin{array}{ll}
F_{2} & -M_{2}
\end{array}\right] .
$$

Taking into account the expression (2.33) for conditional densities, the condition (3.7) implies

$$
\left\|\left[\begin{array}{ll}
F_{s 1} & -M_{s 1}
\end{array}\right]\left[\begin{array}{l}
y \\
x
\end{array}\right]\right\|^{2}=\left\|\left[\begin{array}{ll}
F_{1} & -M_{1}
\end{array}\right]\left[\begin{array}{l}
y \\
x
\end{array}\right]\right\|^{2}+C(y)
$$

for all vectors $\left[y^{T} x^{T}\right]^{T}$ belonging to the right null space of the matrices in (3.16), where $C(y)$ denotes an additive factor depending only on $y$. Consequently, there exist two matrices $U_{1}$ and $U_{12}$, with $U_{1}$ invertible, such that

$$
\left[\begin{array}{cc}
U_{1} & U_{12} \\
0 & U_{2}
\end{array}\right]\left[\begin{array}{cc}
F_{s 1} & -M_{s 1} \\
F_{s 2} & -M_{s 2}
\end{array}\right]=\left[\begin{array}{cc}
F_{1} & -M_{1} \\
F_{2} & -M_{2}
\end{array}\right]
$$

Since $A$ is invertible, this implies that the observation $o_{s}$ has dimension greater than or equal to $o$, so that $o$ is minimal. The identity (3.19) indicates also that two minimal sufficient observations are necessarily related by a left multiplication with an invertible matrix $K$. 
This is due to the fact that if $o_{s}$ is minimal, its canonical decomposition (3.15a) does not include $F_{s 3}$ and $F_{s 4}$ blocks.

To gain some intuition about the concept of minimal observation, consider a minimal observation of the form (2.1), where $x$ is assumed estimable. Then $A$ has both full row and column rank, so that it is invertible, and according to (2.41), $\hat{x}_{M L}=A^{-1} E y$ is the ML estimate of $x$. Without loss of information, we can premultiply (2.1) by $A^{-1}$, which yields

$$
\hat{x}_{M L}=x+G u
$$

with $G=A^{-1} B$, so that $P=G G^{T}$ is the error covariance of the ML estimate. Thus, in this case, a minimal observation represents just a way of coding the ML estimate and error covariance of $x$ without including any extraneous information. More generally, when $x$ is not estimable, a minimal observation represents just a code for the ML estimate and error variance of its estimable part $z=A x$.

The identities (3.12) show that once a minimal sufficient observation has been constructed, it contains no useful information about the constraint space $\mathcal{S}(o)$ and likelihood function $L(o)$ of the original observation $o$. The reduction primitive described below provides a mechanism for constructing minimal sufficient observations, while at the same time keeping track of the constraint space and likelihood function of $o$.

Definition 3.4 (reduction and coreduction) Given an observation o in the canonical form (2.2)-(2.4), the reduced observation $R\{o\}$ and its complement, the coreduction $\bar{R}\{o\}$, are given by

$$
\begin{array}{ll}
R\{o\}: & {\left[\begin{array}{l}
F_{1} \\
F_{2}
\end{array}\right] y=\left[\begin{array}{l}
M_{1} \\
M_{2}
\end{array}\right] x+\left[\begin{array}{c}
u_{1} \\
0
\end{array}\right]} \\
\bar{R}\{o\}: & {\left[\begin{array}{l}
F_{3} \\
F_{4}
\end{array}\right] y=\left[\begin{array}{c}
u_{3} \\
0
\end{array}\right] .}
\end{array}
$$

Combining Lemmas 2.1 and 3.2, we obtain the following result, which justifies our choice of reduction primitive.

\section{Theorem 3.1}

1) $R\{o\}$ is a minimal sufficient observation for o, and

2) the constraint space $\mathcal{S}(o)$ and likelihood function L(o) satisfy

$$
\mathcal{S}(o)=\mathcal{S}(\bar{R}\{o\}) \quad L(o)=L(\bar{R}\{o\}) .
$$

Thus, the reduction operation completely decouples the ML estimation of $x$ from the problem of computing the constraint space and likelihood function of $o$ : the minimal observation $R\{o\}$ contains all the information necessary for the ML estimation of $x$, whereas the 
coreduction $\bar{R}\{o\}$ contains all the information concerning the constraint space and likelihood function of $o$.

Finally, note that although the primitives $R\{$.$\} and \bar{R}\{$.$\} are represented by different$ symbols, because they can both be implemented with the decomposition algorithm of Lemma 2.1 , it can be argued they form only one primitive. However, from a computational point of view, it is preferable to implement $\bar{R}\{$.$\} and R\{$.$\} as distinct entities. Indeed, as indicated$ by the Remark 2) following the proof of Lemma 2.1, given an observation $o$ of the form (2.1), the generation of $\bar{R}\{o\}$ requires only the first step of the procedure employed to find $R\{o\}$. Specifically, if $L$ is a matrix whose rows form a basis of the left null space of $A$, the coreduction $\bar{R}\{o\}$ takes the form (2.16). Thus, the full decomposition procedure of Lemma 2.1 needs to be applied only if we seek to compute both $R\{o\}$ and $\bar{R}\{o\}$.

\section{Mutation and Other Macros}

In addition to the three primitives: $\wedge, R\{\},. \bar{R}\{$.$\} previously introduced, we need to introduce$ a fourth primitive whose effect is to change the status of a vector inside an observation from known to unknown, or vice-versa. This primitive will be useful to formulate the concept of conditional observation, where certain vectors need to be treated temporarily as known, even though they are actually unknown.

Definition 3.5 Given an observation of the form

$$
o: \quad E y=\left[\begin{array}{ll}
A_{0} & A_{1}
\end{array}\right]\left[\begin{array}{l}
x_{0} \\
x_{1}
\end{array}\right]+B u
$$

the observation

$$
M_{x_{1}}\{o\}: \quad\left[\begin{array}{ll}
E & -A_{1}
\end{array}\right]\left[\begin{array}{c}
y \\
x_{1}
\end{array}\right]=A_{0} x_{0}+B u
$$

obtained by changing the status of the vector $x_{1}$ from unknown to known, is said to be a mutation of $x_{1}$ inside o. Similarly, given

$$
o^{\prime}: \quad\left[\begin{array}{ll}
E_{0} & E_{1}
\end{array}\right]\left[\begin{array}{l}
y_{0} \\
y_{1}
\end{array}\right]=A x+B u
$$

the observation

$$
M_{y_{1}}\left\{o^{\prime}\right\}: \quad E_{0} y_{0}=\left[\begin{array}{cc}
A & -E_{1}
\end{array}\right]\left[\begin{array}{c}
x \\
y_{1}
\end{array}\right]+B u
$$

generated by changing the status of $y_{1}$ from known to unknown constitutes a mutation of $y_{1}$ inside $o^{\prime}$.

Clearly, two consecutive mutations of the same vector yield the original observation. Consider now an observation of the form (3.24), and suppose we are no longer interested in the vector $x_{0}$ and just seek to "extract" the information about $y$ and $x_{1}$ contained in (3.24). This amounts to computing the marginal density $\mathbf{p}\left(y, x_{1}\right)$ and constraint space $\mathcal{C}_{1}$ 
for $\left[\begin{array}{ll}y^{T} & x_{1}^{T}\end{array}\right]^{T}$ from the pair $\left(\mathbf{p}\left(y, x_{0}, x_{1}\right), \mathcal{C}\right)$ associated to $o$. Referring to the property $(3.23)$ of the coreduction $\bar{R}\{$.$\} , we see it provides a mechanism for extracting the marginal density$ and constraint space associated to the measurement vector $y$. If we want to include the vector $x_{1}$ in the marginal computation, we need only to change its status and view it as a known vector before applying the $\bar{R}\{$.$\} primitive. After \bar{R}\{$.$\} has been applied, we can of$ course convert back $x_{1}$ to its original "unknown" status. This motivates the construction of the following two macros, which are expressed entirely in terms of the four primitives already introduced.

Definition 3.6 (conditional observation, extraction) Given an observation of the form (3.24), the conditional observation of o given $x_{1}$, and the extraction of $x_{1}$ from $o$, are defined respectively as

$$
\begin{aligned}
o \mid x_{1} & =R_{\circ} M_{x_{1}}\{o\} \\
X_{x_{1}}\{o\} & =M_{x_{1}} \bar{R}_{\circ} M_{x_{1}}\{o\},
\end{aligned}
$$

where "" denotes the composition of maps.

Taking into account the comment preceding (2.16), we see that if $L$ is a matrix whose rows form a basis of the left null space of $A_{0}, X_{x_{1}}\{o\}$ can be expressed as

$$
X_{x_{1}}\{o\}: \quad L E y=L A_{1} x_{1}+L B u .
$$

Thus, in the context of statistical models containing mixed deterministic/stochastic information, the macros $X_{x_{1}}\{o\}$ and $o \mid x_{1}$ perform respectively the computation of marginal and conditional densities and constraint spaces.

\section{Properties of the primitives and macros}

This subsection describes several useful properties of the primitives and macros we have introduced. We begin with simple formulas involving a single observation. Their proof is immediate.

Lemma 3.3 The following formulas hold:

$$
\begin{aligned}
& o=R\{o\} \wedge \bar{R}\{o\} \\
& o=X_{x_{1}}\{o\} \wedge M_{x_{1}}\left\{o \mid x_{1}\right\},
\end{aligned}
$$

where in (3.306), we assume o has the form (3.24).

The extracted observation $X_{x_{1}}\{o\}$ has the following properties.

\section{Theorem 3.2}

1) Given an observation of the form (3.24), the $M L$ estimates of the estimable part of $x_{1}$ based on o and $X_{x_{1}}\{o\}$ are the same. 
2) The extraction operation preserves the information contained in o concerning the measurement vector $y$, in the sense that

$$
\bar{R}_{\circ} X_{x_{1}}\{o\}=\bar{R}\{o\} .
$$

In particular, we have

$$
\mathcal{S}(o)=\mathcal{S}\left(X_{x_{1}}\{o\}\right) \quad L(o)=L\left(X_{x_{1}}\{o\}\right) .
$$

Proof: Consider the decomposition (3.30b) of $o$. Since $o \mid x_{1}$ is by definition a minimal observation, according to the Lemma 3.2 , its marginal density with respect to the pair $\left(y, x_{1}\right)$ is a constant, and $o \mid x_{1}$ does not impose any constraint on $\left(y, x_{1}\right)$. But applying the mutation operation $M_{x_{1}}$ does not affect either the joint density of $y$ and $x_{1}$ or their constraint space. Thus, in the decomposition $X_{x_{1}}\{o\} \wedge M_{x_{1}}\left\{o \mid x_{1}\right\}$ of observation $o$, the second component does not provide any information about $x_{1}$. This proves the first statement of of the theorem.

Also, since $\bar{R}\{o\}$ just encodes the constraint space and likelihood function of $y$, when applying $\bar{R}$ to the decomposition $X_{x_{1}}\{o\} \wedge M_{x_{1}}\left\{o \mid x_{1}\right\}$, we can ignore the second observation. This proves formula (3.31). Then, (3.32) is just a consequence of (3.31) and (3.23).

We now move to properties involving two observations. Note first that for an arbitrary vector $z$, we have the obvious identity

$$
M_{z}\left\{o_{1} \wedge o_{2}\right\}=M_{z}\left\{o_{1}\right\} \wedge M_{z}\left\{o_{2}\right\} .
$$

Next, observe that the presence of the coupling parameter vector $x_{c}$ in expression (3.2) has the effect of complicating the properties of the combinator $\wedge$. Thus, it is of interest introduce the absence of coupling vector $x_{c}$ as a special property. On the other hand, the presence of a coupling measurement $y_{c}$ causes no particular trouble, as will be seen below.

Definition 3.7 (independent observations) Two observations $o_{1}$ and $o_{2}$ with the structure (3.1a)-(3.1b) are said to be independent, which is denoted as

$$
o_{1} \perp o_{2}
$$

if they do not include any unknown coupling vector $x_{c}$, or, more generally, if, for $i=1$ or 2 , the extracted observation $X_{x_{c}}\left\{o_{i}\right\}$ does not depend on $x_{c}$, i.e.

$$
X_{x_{c}}\left\{o_{i}\right\}=\bar{R}\left\{o_{i}\right\} .
$$

The property (3.34) is apparently weaker than the absence of coupling, since it only requires that $o_{i}$ for $i=1$ or 2 should convey no information about the coupling vector $x_{c}$. However, as the following lemma indicates, when (3.34) is satisfied, the observations $o_{1}$ and $\mathrm{o}_{2}$ can be reparametrized so as to make them decoupled. 
Lemma 3.4 If an observation on of the form (3.1a) satisfies (3.34), there exists a matrix $T$ such that $o_{1}$ can be rewritten in terms of the new vector

$$
x_{1}^{\prime}=x_{1}+T x_{c},
$$

as

$$
o_{1}: \quad\left[\begin{array}{ll}
E_{11} & E_{1 c}
\end{array}\right]\left[\begin{array}{l}
y_{1} \\
y_{c}
\end{array}\right]=A_{11} x_{1}^{\prime}+B_{1} u_{1}
$$

which is now decoupled from the observation $\mathrm{O}_{2}$ of (3.16).

Proof: If $X_{x_{c}}\left\{o_{1}\right\}$ is independent of $x_{c}$, the expression (3.29) for the extraction operation indicates that if $L$ is a basis of the left null space of $A_{11}$, we must have

$$
L A_{1 c}=0
$$

i.e. $L$ must be in the left null space of $A_{1 c}$. Consequently, the column space of $A_{1 c}$ is contained in the column space of $A_{11}$, so that we can find a matrix $T$ such that

$$
A_{1 c}=A_{11} T .
$$

Substituting this relation inside (3.1a), and defining $x_{1}^{\prime}$ as in (3.35a), gives (3.35b).

In general, because of the presence of the coupling vector $x_{c}$, the reduction $R\left\{o_{1} \wedge o_{2}\right\}$, likelihood function $L\left(o_{1} \wedge o_{2}\right)$ and constraint space $\mathcal{S}\left(o_{1} \wedge o_{2}\right)$ cannot be expressed purely in terms of $\left(R\left\{o_{i}\right\}, L\left(o_{i}\right), \mathcal{S}\left(o_{i}\right)\right)$ with $i=1,2$. However, when $o_{1}$ and $o_{2}$ are independent, we have the following expressions.

Lemma 3.5 If $o_{1} \perp o_{2}$, the combined observation $o=o_{1} \wedge o_{2}$ satisfies

$$
\begin{aligned}
& R\left\{o_{1} \wedge o_{2}\right\}=R\left\{o_{1}\right\} \wedge R\left\{o_{2}\right\} \\
& \bar{R}\left\{o_{1} \wedge o_{2}\right\}=\bar{R}\left\{o_{1}\right\} \wedge \bar{R}\left\{o_{2}\right\} .
\end{aligned}
$$

In particular, we have

$$
\begin{aligned}
\mathcal{S}^{\perp}(o) & =\mathcal{S}^{\perp}\left(o_{1}\right)+\mathcal{S}^{\perp}\left(o_{2}\right) \\
L(o) & =L\left(o_{1}\right) L\left(o_{2}\right)
\end{aligned}
$$

where + denotes the (not necessarily direct) sum of vector spaces. Furthermore, if there is no coupling measurement $y_{c}$, the sum in (3.39a) becomes direct.

Proof: Select a coordinate system such that $o_{1}$ and $o_{2}$ have no coupling vector $x_{c}$. Next, observe that the canonical decomposition of an observation given in Lemma 2.1 depends only on the matrix pair $(A, B)$. Since the noises $u_{1}$ and $u_{2}$ are independent and $o_{1}$ and $o_{2}$ have no coupling vector, the canonical decomposition (2.2)-(2.4) for $o_{1} \wedge o_{2}$ can be obtained by reducing and coreducing separately $o_{1}$ and $o_{2}$, and then forming $R\left\{o_{1}\right\} \wedge R\left\{o_{2}\right\}$ and

$\operatorname{RR} \mathrm{n}^{\circ} 2088$ 
$\bar{R}\left\{o_{1}\right\} \wedge \bar{R}\left\{o_{2}\right\}$, which justifies (3.38a)-(3.38b). Then, using the representations (2.17) and $(2.32)$ of $\mathcal{S}(o)$ and $L(o)$, gives $(3.39 \mathrm{a})-(3.39 \mathrm{~b})$.

The property (3.39a), which is expressed in terms of the parity spaces of $o_{1}$ and $o_{2}$, can be written equivalently in terms of their constraint spaces as

$$
\mathcal{S}(o)=\mathcal{S}\left(o_{1}\right) \cap \mathcal{S}\left(o_{2}\right) .
$$

Furthermore, when the observations $o_{1}$ and $o_{2}$ do not contain a common measurement component $y_{c}$, the parity check tests for $o_{1}$ and $o_{2}$ concern different measurement vectors $y_{1}$ and $y_{2}$, so that the sum in (3.39a) can be replaced by a direct sum.

As a corollary of the above lemma, we have the following result.

Corollary 3.1 If $o_{1} \perp o_{2}$, then for any unknown vector $z$,

$$
X_{z}\left\{o_{1} \wedge o_{2}\right\}=X_{z}\left\{o_{1}\right\} \wedge X_{z}\left\{o_{2}\right\} .
$$

\section{E Recursion Principle}

We have now at our disposal all the tools necessary to develop recursive algorithms for computing ML estimates, and to evaluate the constraint set and likelihood function associated

to a stream of observations. The algorithms that we shall derive rely on the recursion scheme described below, which is a consequence of Theorems 3.1 and 3.2 .

Theorem 3.3 Consider the observations

$$
\begin{aligned}
& o_{1}: \quad E_{11} y_{1}+E_{1 c} y_{c}=A_{11} x_{1}+A_{1 c} x_{c}+B_{1} u_{1} \\
& o_{2}: \\
& E_{2 c} y_{c}+E_{22} y_{2}=A_{2 c} x_{c}+A_{22} x_{2}+B_{2} u_{2},
\end{aligned}
$$

where $u_{1}$ and $u_{2}$ are two zero-mean independent Gaussian vectors with unit covariance. Then

$$
R_{\circ} X_{x_{2}}\left\{o_{1} \wedge o_{2}\right\}=R_{\circ} X_{x_{2}}\left\{R_{\circ} X_{x_{c}}\left\{o_{1}\right\} \wedge o_{2}\right\}
$$

and

$$
\bar{R}\left\{o_{1} \wedge o_{2}\right\}=\bar{R}_{\circ} X_{x_{2}}\left\{R_{\circ} X_{x_{c}}\left\{o_{1}\right\} \wedge o_{2}\right\} \wedge \bar{R}_{\circ} X_{x_{c}}\left\{o_{1}\right\}
$$

which in turn implies

$$
\begin{aligned}
\mathcal{S}^{\perp}\left(o_{1} \wedge o_{2}\right) & =\mathcal{S}^{\perp}\left(\bar{R}_{\circ} X_{x_{2}}\left\{R_{\circ} X_{x_{c}}\left\{o_{1}\right\} \wedge o_{2}\right\}\right)+\mathcal{S}^{\perp}\left(\bar{R}_{\circ} X_{x_{c}}\left\{o_{1}\right\}\right) \\
L\left(o_{1} \wedge o_{2}\right) & =L\left(\bar{R}_{\circ} X_{x_{2}}\left\{R_{\circ} X_{x_{c}}\left\{o_{1}\right\} \wedge o_{2}\right\}\right) L\left(\bar{R}_{\circ} X_{x_{c}}\left\{o_{1}\right\}\right)
\end{aligned}
$$

where the sum in (3.45a) can be replaced by a direct sum whenever $o_{1}$ and $o_{2}$ do not contain a common measurement $y_{c}$. 
Proof: We first derive (3.43). Decomposing $o_{1}$ as in (3.30a), and observing that $R\left\{o_{1}\right\} \wedge o_{2}$ and $\bar{R}\left\{o_{1}\right\}$ are independent, the property (3.41) gives

$$
X_{x_{2}}\left\{o_{1} \wedge o_{2}\right\}=X_{x_{2}}\left\{R\left\{o_{1}\right\} \wedge o_{2}\right\} \wedge X_{x_{2}} \circ \bar{R}\left\{o_{1}\right\} .
$$

Since $x_{2}$ does not appear in $o_{1}, X_{x_{2}} \circ \bar{R}\left\{o_{1}\right\}=\bar{R}\left\{o_{1}\right\}$. We can thus apply (3.38a), which yields

$$
\begin{aligned}
R\left\{X_{x_{2}}\left\{R\left\{o_{1}\right\} \wedge o_{2}\right\} \wedge \bar{R}\left\{o_{1}\right\}\right\} & =R_{\circ} X_{x_{2}}\left\{R\left\{o_{1}\right\} \wedge o_{2}\right\} \wedge R_{\circ} \bar{R}\left\{o_{1}\right\} \\
& =R_{\circ} X_{x_{2}}\left\{R\left\{o_{1}\right\} \wedge o_{2}\right\} .
\end{aligned}
$$

Now, according to (3.30b), o can be decomposed as

$$
o_{1}=X_{x_{c}}\left\{o_{1}\right\} \wedge M_{x_{c}}\left\{o_{1} \mid x_{c}\right\},
$$

where $X_{x_{c}}\left\{o_{1}\right\}$ and $M_{x_{c}}\left\{o_{1} \mid x_{c}\right\}$ are independent since $M_{x_{c}}\left\{o_{1} \mid x_{c}\right\}$ does not convey any information about $x_{c}$. Applying the reduction operation $R$ to both sides, and noting that $R_{\circ} M_{x_{c}}\left\{o_{1} \mid x_{c}\right\}$ is independent from observation $R_{\circ} X_{x_{c}}\left\{o_{1}\right\} \wedge o_{2}$, we can apply again (3.41). This gives

$$
\begin{aligned}
X_{x_{2}}\left\{\left(R_{\circ} X_{x_{c}}\left\{o_{1}\right\} \wedge o_{2}\right) \wedge R_{\circ} M_{x_{c}}\left\{o_{1} \mid x_{c}\right\}\right\} & =X_{x_{2}}\left\{\left(R_{\circ} X_{x_{c}}\left\{o_{1}\right\} \wedge o_{2}\right)\right\} \wedge X_{x_{2}} \circ R_{\circ} M_{x_{c}}\left\{o_{1} \mid x_{c}\right\} \\
& =X_{x_{2}}\left\{\left(R_{\circ} X_{x_{c}}\left\{o_{1}\right\} \wedge o_{2}\right)\right\} .
\end{aligned}
$$

Then, combining (3.47) and (3.49), we get (3.43).

Next, we prove (3.44). Using (3.46) and then (3.49) yields

$$
\begin{aligned}
X_{x_{2}}\left\{o_{1} \wedge o_{2}\right\} & =X_{x_{2}}\left\{R\left\{o_{1}\right\} \wedge o_{2}\right\} \wedge X_{x_{2} \circ} \bar{R}\left\{o_{1}\right\} \\
& =X_{x_{2}}\left\{R\left\{o_{1}\right\} \wedge o_{2}\right\} \wedge \bar{R}\left\{o_{1}\right\} \\
& =X_{x_{2}}\left\{R_{\circ} X_{x_{c}}\left\{o_{1}\right\} \wedge o_{2} \wedge R_{\circ} M_{x_{c}}\left\{o_{1} \mid x_{c}\right\}\right\} \wedge \bar{R}\left\{o_{1}\right\} \\
& =X_{x_{2}}\left\{R_{\circ} X_{x_{c}}\left\{o_{1}\right\} \wedge o_{2}\right\} \wedge \bar{R}\left\{o_{1}\right\} .
\end{aligned}
$$

But

$$
\bar{R}_{\circ} X_{x_{2}}\left\{o_{1} \wedge o_{2}\right\}=\bar{R}\left\{o_{1} \wedge o_{2}\right\}
$$

and since observations $X_{x_{2}}\left\{R_{\circ} X_{x_{c}}\left\{o_{1}\right\} \wedge o_{2}\right\}$ and $\bar{R}\left\{o_{1}\right\}$ are independent, by using (3.38b), we find

$$
\begin{aligned}
\bar{R}\left\{X_{x_{2}}\left\{R_{\mathrm{o}} X_{x_{c}}\left\{o_{1}\right\} \wedge o_{2}\right\} \wedge \bar{R}\left\{o_{1}\right\}\right\} & =\bar{R}_{\mathrm{o}} X_{x_{2}}\left\{R_{\mathrm{o}} X_{x_{c}}\left\{o_{1}\right\} \wedge o_{2}\right\} \wedge \bar{R}\left\{o_{1}\right\} \\
& =\bar{R}_{\mathrm{o}} X_{x_{2}}\left\{R_{\mathrm{o}} X_{x_{c}}\left\{o_{1}\right\} \wedge o_{2}\right\} \wedge \overline{R_{\mathrm{o}}} X_{x_{c}}\left\{o_{1}\right\}
\end{aligned}
$$

where (3.52) uses the identity $\bar{R}_{\circ} X_{x_{c}}=\bar{R}$. Combining (3.50)-(3.52) gives (3.44).

Thus our approach consists of estimating vectors in the order in which they arise in observations, and then discard these vectors for the purpose of processing subsequent observations where they do not appear. The constraint space $\mathcal{S}(o)$ is obtained by juxtaposition of the old constraints with new constraints for the latest observations. $L(o)$ is obtained by multiplying the old likelihood function with a term reflecting the new information, i.e. the "innovation", contained in the most recent measurement. 


\section{Filtering and Smoothing of Descriptor Systems and Application to Failure Detection}

To illustrate the primitives and other macros introduced in the previous section, we solve the filtering and smoothing problems for linear descriptor systems, and then apply our results to a failure detection problem. Linear descriptor systems admit dynamics of the form

$$
d_{k+1}: \quad 0=-E_{k+1} x_{k+1}+A_{k} x_{k}+B_{k} u_{k},
$$

with observations

$$
m_{k+1}: \quad y_{k+1}=C_{k+1} x_{k+1}+D_{k} u_{k}
$$

for $0 \leq k \leq N-1$, where $u_{k}$ is a white Gaussian noise such that $u_{k} \sim \mathcal{N}(0, I)$. The case $E_{k+1}=I$ corresponds to the usual state-space models, but $E_{k+1}$ need not be invertible in general. In fact, the matrices $E_{k+1}$ and $A_{k}$ are not even required to be square, and the size of the state $x_{k}$ can change with $k$. The presence of deterministic information is reflected here by the fact that the dynamics (4.1) may contain fixed algebraic constraints between entries of $x_{k+1}$ and $x_{k}$, and the covariance $D_{k} D_{k}^{T}$ of the observation noise may not be invertible, thus resulting in a singular estimation problem.

The filtering problem for descriptor systems has been examined by several researchers [12], [13], [14], [7]. For the case when $x_{k}$ is estimable from past observations, explicit recursions for the optimum filter and its associated Riccati equation are given in [13], which contains also an analysis of the stady-state convergence of the optimum filter. Square-root implementations of the descriptor Kalman filter were proposed in [15] and [14]. The case where $x_{k}$ is not estimable from past observations (but may be estimable from past and future observations combined) is considered in [7], where the filtering and smoothing formulas were expressed for the first time in terms of the reduction and extraction primitives employed here. However, because a probabilistic interpretation of the primitives was not yet available, the solution of the filtering problem presented in [7] does not include the generation of an innovations process, or parity checks for evaluating the model validity in function of the received observations. Both of these items have important practical applications, since as will be shown below the computation of the likelihood function associated to model (4.1)-(4.2) relies on the innovations process. Furthermore, a wide class of failure detection algorithms [16], [17] relies on parity check tests of the type discussed here.

When considering the system (4.1)-(4.2), we make no distinction between dynamics and measurements, and view all linear relations relating different random variables as observations for these variables. From this point of view, it is convenient to combine the dynamics and measurement equations (4.1)-(4.2) into a single observation $o_{k+1}=d_{k+1} \wedge m_{k+1}$ of the form

$$
o_{k+1}: \quad z_{k+1}=\left[\begin{array}{c}
0 \\
y_{k+1}
\end{array}\right]=\left[\begin{array}{c}
-E_{k+1} \\
C_{k+1}
\end{array}\right] x_{k+1}+\left[\begin{array}{c}
A_{k} \\
0
\end{array}\right] x_{k}+\left[\begin{array}{c}
B_{k} \\
D_{k}
\end{array}\right] u_{k}
$$


for $0 \leq k \leq N-1$. The information available about the initial and final states is assumed to have the separable form

$$
\begin{aligned}
& o_{0}: \quad z_{0}=-E_{0} x_{0}+B_{-1} u_{-1} \\
& o_{N+1}: z_{N+1}=A_{N} x_{N}+B_{N} u_{N} \text {. }
\end{aligned}
$$

\section{A Filtering}

Let

$$
P_{k}=\bigwedge_{j=0}^{k} o_{j}
$$

be the observation obtained by combining all past observations up to time $k$. Then, the filtered observation describing the information about $x_{k}$ contained in $P_{k}$, the constraint space of $P_{k}$, and its likelihood function, are denoted respectively by

$$
\begin{array}{cc}
\hat{o}_{k \mid k}^{f}= & R_{\circ} X_{x_{k}}\left\{P_{k}\right\} \\
\mathcal{S}_{k}=\mathcal{S}\left\{P_{k}\right\} & L_{k}=L\left(P_{k}\right) .
\end{array}
$$

Applying the recursion principle of Theorem 3.3, we obtain the Kalman filtering recursions

$$
\begin{aligned}
\hat{o}_{k+1 \mid k+1}^{f} & =R_{\circ} X_{x_{k+1}}\left\{\hat{o}_{k \mid k}^{f} \wedge o_{k+1}\right\} \\
\mathcal{S}_{k+1}^{\perp} & =\mathcal{S}^{\perp}\left(\bar{R}_{\circ} X_{x_{k+1}}\left\{\hat{o}_{k \mid k}^{f} \wedge o_{k+1}\right\}\right) \oplus \mathcal{S}_{k}^{\perp} \\
L_{k+1} & =L\left(\bar{R}_{\circ} X_{x_{k+1}}\left\{\hat{o}_{k \mid k}^{f} \wedge o_{k+1}\right\}\right) L_{k}
\end{aligned}
$$

with initial conditions

$$
\hat{o}_{0 \mid 0}^{f}=R\left\{o_{0}\right\} \quad \mathcal{S}_{0}=\mathcal{S}\left(\bar{R}\left\{o_{0}\right\}\right) \quad L_{0}=L\left(\bar{R}\left\{o_{0}\right\}\right),
$$

where $\oplus$ denotes the direct sum of vector spaces. These recursions, which obviously constitute a rather unusual form of the Kalman filter, are totally expressed in terms of the primitives and macros introduced in Section 3. To interpret the expressions obtained for $\mathcal{S}_{k}^{\perp}$ and $L_{k}$, note from the definition (3.22) of $\bar{R}\{$.$\} that the observation \bar{R}_{\mathrm{o}} X_{x_{k+1}}\left\{\hat{o}_{k \mid k}^{f} \wedge o_{k+1}\right\}$ can be decomposed as

$$
\left[\begin{array}{l}
\nu_{k+1} \\
\pi_{k+1}
\end{array}\right]=\left[\begin{array}{c}
w_{k+1} \\
0
\end{array}\right]
$$

where $w_{k+1} \sim \mathcal{N}(0, I)$. The vectors $\nu_{k+1}$ and $\pi_{k+1}$ correspond respectively to the innovations and parity checks of the given model, and with respect to these vectors, the recursions (4.7b)(4.7c) take the form

$$
\begin{aligned}
\mathcal{S}_{k+1}^{\perp} & =\left\{y_{j}, 0 \leq j \leq k+1: \pi_{k+1}=0\right\}^{\perp} \oplus \mathcal{S}_{k}^{\perp} \\
L_{k+1} & =\exp -\frac{1}{2}\left\|\nu_{k+1}\right\|^{2} L_{k}
\end{aligned}
$$


so that, as for nonsingular filtering problems, the log-likelihood function $l_{k}=-\ln L_{k}$ is obtained by summing the squared innovations.

Interpretation: The algorithm (4.7a)-(4.7c) is quite compact and constitutes by itself a complete implementation of the Kalman filter. However, for interpretation purposes, it can be transcribed into a form closer to the usual Kalman filter if we assume that $x_{k}$ is estimable from $P_{k}$, so that $\left[-E_{k}^{T} C_{k}^{T}\right]^{T}$ has full rank for all $k$. In this case, $\hat{o}_{k \mid k}^{f}$ can be written as

$$
\hat{o}_{k \mid k}^{f}: \quad \hat{x}_{k \mid k}^{f}=x_{k}+\Sigma_{k \mid k}^{f 1 / 2} v_{k}
$$

where $\hat{x}_{k \mid k}^{f}$ and $\Sigma_{k \mid k}^{f}$ denote respectively the ML estimate and error covariance matrix of $x_{k}$ given $P_{k}$, and $v_{k} \sim \mathcal{N}(0, I)$ is independent of the model noise $u_{k}$. Also, given an arbitary symmetric nonnegative definite matrix $\Sigma, \Sigma^{1 / 2}$ denotes one of its square-roots. Then the Kalman filtering algorithm (4.7a)-(4.7c) can be broken into two steps.

(i) Time update: We consider $\hat{o}_{k \mid k}^{f} \wedge d_{k+1}$, which takes the form

$$
\left[\begin{array}{c}
\hat{x}_{k \mid k}^{f} \\
0
\end{array}\right]=\left[\begin{array}{c}
0 \\
-E_{k+1}
\end{array}\right] x_{k+1}+\left[\begin{array}{c}
I \\
A_{k}
\end{array}\right] x_{k}+\left[\begin{array}{cc}
\Sigma_{k \mid k}^{f 1 / 2} & 0 \\
0 & B_{k}
\end{array}\right]\left[\begin{array}{c}
v_{k} \\
u_{k}
\end{array}\right]
$$

The predicted observation $\hat{o}_{k+1 \mid k}^{f} \triangleq X_{x_{k+1}}\left\{\hat{o}_{k \mid k}^{f} \wedge d_{k+1}\right\}$ is obtained by premultiplying (4.12) by $\left[-A_{k} I\right]$, which yields

$$
\hat{o}_{k+1 \mid k}^{f}: \quad-A_{k} \hat{x}_{k \mid k}^{f}=-E_{k+1} x_{k+1}+B_{k} u_{k}-A_{k} \Sigma_{k \mid k}^{f 1 / 2} v_{k} .
$$

Since extractions do not change observation constraint spaces or likelihood functions, we have

$$
\begin{aligned}
& \mathcal{S}_{k+1 \mid k} \triangleq \mathcal{S}\left(\hat{o}_{k+1 \mid k}^{f}\right)=\mathcal{S}_{k} \\
& L_{k+1 \mid k} \triangleq L\left(\hat{o}_{k+1 \mid k}^{f}\right)=L_{k} .
\end{aligned}
$$

(ii) Measurement update: Consider now the observation $\hat{o}_{k+1 \mid k}^{f} \wedge m_{k+1}$ :

$$
\left[\begin{array}{c}
-A_{k} \hat{x}_{k \mid k}^{f} \\
y_{k+1}
\end{array}\right]=\left[\begin{array}{c}
-E_{k+1} \\
C_{k+1}
\end{array}\right] x_{k+1}+\left[\begin{array}{cc}
B_{k} & -A_{k} \Sigma_{k \mid k}^{f 1 / 2} \\
D_{k} & 0
\end{array}\right]\left[\begin{array}{c}
u_{k} \\
v_{k}
\end{array}\right] .
$$

The transformation matrix $S_{k+1}$ needed to bring this observation to the canonical form $(2.2)-(2.4)$ can be partitioned as

$$
S_{k+1}=\left[\begin{array}{c}
S_{k+11} \\
S_{k+12} \\
S_{k+13} \\
S_{k+14}
\end{array}\right]
$$


With this notation, the reduced observation $\hat{o}_{k+1 \mid k+1}^{f}=R\left\{\hat{o}_{k+1 \mid k}^{f} \wedge m_{k+1}\right\}$ takes the form

$$
\left[\begin{array}{c}
S_{k+11} \\
S_{k+2} 2
\end{array}\right]\left[\begin{array}{c}
-A_{k} \hat{x}_{k \mid k}^{f} \\
y_{k+1}
\end{array}\right]=M_{k+1} x_{k+1}+\left[\begin{array}{c}
v_{k+1} \\
0
\end{array}\right],
$$

with $v_{k+1} \sim \mathcal{N}(0, I)$. Because of the estimability of $x_{k+1}, M_{k+1}$ is invertible, and upon left multiplication of (4.17) by

$$
M_{k+1}^{-1}=\left[\begin{array}{ll}
Q_{k+11} & Q_{k+12}
\end{array}\right]
$$

we obtain

$$
\hat{x}_{k+1 \mid k+1}^{f}: \quad \hat{x}_{k+1 \mid k+1}^{f}=x_{k+1}+\Sigma_{k+1 \mid k+1}^{f 1 / 2} v_{k+1}
$$

with

$$
\begin{aligned}
\hat{x}_{k+1 \mid k+1}^{f} & =M_{k+1}^{-1}\left[\begin{array}{c}
S_{k+11} \\
S_{k+12}
\end{array}\right]\left[\begin{array}{c}
-A_{k} \hat{x}_{k \mid k}^{f} \\
y_{k+1}
\end{array}\right] \\
\Sigma_{k+1 \mid k+1}^{f 1 / 2} & =Q_{k+11} .
\end{aligned}
$$

Similarly, $\bar{R}\left\{\hat{o}_{k+1 \mid k}^{f} \wedge o_{k+1}\right\}$ is given by

$$
\left[\begin{array}{c}
S_{k+13} \\
S_{k+14}
\end{array}\right]\left[\begin{array}{c}
-A_{k} \hat{x}_{k \mid k}^{f} \\
y_{k+1}
\end{array}\right]=\left[\begin{array}{c}
\nu_{k+1} \\
\pi_{k+1}
\end{array}\right]=\left[\begin{array}{c}
w_{k+1} \\
0
\end{array}\right],
$$

so that the innovations $\nu_{k+1}$ and parity check vector $\pi_{k+1}$ are linear combinations of $\hat{x}_{k \mid k}^{f}$ and $y_{k+1}$.

Note that the above discussion merely provides a step by step account of the operations appearing in the Kalman filtering algorithm (4.7a)-(4.7c). However our goal is ultimately to hide all implementation details of this type by focusing on the larger picture provided by the primitives and macros we have introduced.

\section{B Two-filter Smoother}

To discuss two-filter implementations of the smoother associated to the model (4.1)-(4.2), it is convenient to construct the backward Kalman filter for this model. Thus, let

$$
F_{k}=\bigwedge_{j=k}^{N+1} o_{j}
$$

be the combination of all observations in the future of $k$. Then the backward predicted observation $\hat{o}_{k \mid k+1}^{b}$ describing the information about $x_{k}$ contained in $F_{k+1}$ is given by

$$
\hat{o}_{k \mid k+1}^{b}=R_{\circ} X_{x_{k}}\left\{F_{k+1}\right\} .
$$


According to Theorem 3.2, it satisfies the backward Kalman recursion (in predicted form)

$$
\begin{aligned}
\hat{o}_{k-1 \mid k}^{b} & =R_{\circ} X_{x_{k-1}}\left\{o_{k \mid k+1}^{b} \wedge o_{k}\right\} \\
\hat{o}_{N+1 \mid N+2}^{b} & =R\left\{o_{N+1}\right\} .
\end{aligned}
$$

Then, if

$$
O=\bigwedge_{j=0}^{N+1} o_{j}
$$

represents the combination of all observations, the smoothed observation

$$
\hat{o}_{k}^{s}=R_{\circ} X_{x_{k}}\{O\}
$$

describes the information about $x_{k}$ contained in all observations. Instead of having to process all observations simultaneously, as indicated by the formula (4.26), by observing that $O=$ $P_{k} \wedge F_{k+1}$, we obtain the two-filter smoothing formula

$$
\hat{o}_{k}^{s}=R\left\{\hat{o}_{k \mid k}^{f} \wedge \hat{o}_{k \mid k+1}^{b}\right\} .
$$

Interpretation: The above expression, through its use of estimation primitives and macros, provides a totally self-contained implementation of the two-filter smoother. However, for the sake of consistency with past work, it can be converted to the standard form of this smoother [18], [19] which expresses the smoothed estimate as a weighted combination of the forward and and backward Kalman filtering estimates. To do so, assume that $x_{k}$ is estimable separately from $P_{k}$ and $F_{k+1}$, and that the corresponding forward and backward error covariance matrices $\Sigma_{k \mid k}^{f}$ and $\Sigma_{k \mid k+1}^{b}$ are positive definite. The estimability assumption for $x_{k}$ based on $F_{k+1}$ is quite stringent since it requires that the dynamic matrix $A_{k}$ should be invertible, which is often not true. Under these assumptions, the combined observation $\hat{o}_{k \mid k}^{f} \wedge \hat{o}_{k \mid k+1}^{b}$ can be expressed as

$$
\left[\begin{array}{c}
\hat{x}_{k \mid k}^{f} \\
\hat{x}_{k \mid k+1}^{b}
\end{array}\right]=\left[\begin{array}{c}
I \\
I
\end{array}\right] x_{k}+\left[\begin{array}{cc}
\Sigma_{k \mid k}^{f 1 / 2} & 0 \\
0 & \Sigma_{k \mid k+1}^{b 1 / 2}
\end{array}\right]\left[\begin{array}{c}
u_{k}^{f} \\
u_{k}^{b}
\end{array}\right] .
$$

The transformations which bring it to canonical form (where for convenience the nonzero blocks of $S B V$ in (2.2) are not normalized to the identity) can be selected as

$$
\begin{aligned}
S_{k} & =\left[\begin{array}{cc}
\left(\Sigma_{k \mid k}^{f}\right)^{-1} & \left(\Sigma_{k \mid k+1}^{b}\right)^{-1} \\
I & -I
\end{array}\right] \\
V_{k} & =\left[\begin{array}{cc}
\left(\Sigma_{k}^{s}\right)^{1 / 2} & 0 \\
0 & \left(Q_{k}^{s}\right)^{-1 / 2}
\end{array}\right]\left[\begin{array}{cc}
\left(\Sigma_{k \mid k}^{f}\right)^{-T / 2} & \left(\Sigma_{k \mid k+1}^{b}\right)^{-T / 2} \\
\Sigma_{k \mid k}^{f 1 / 2} & -\Sigma_{k \mid k+1}^{b 1 / 2}
\end{array}\right],
\end{aligned}
$$


with

$$
\begin{aligned}
\left(\Sigma_{k}^{s}\right)^{-1} & =\left(\Sigma_{k \mid k}^{f}\right)^{-1}+\left(\Sigma_{k \mid k+1}^{b}\right)^{-1} \\
Q_{k}^{s} & =\Sigma_{k \mid k}^{f}+\Sigma_{k \mid k+1}^{b} .
\end{aligned}
$$

Note that $V_{k}$ is orthonormal. This gives

$$
\hat{o}_{k}^{s}: \quad\left(\Sigma_{k}^{s}\right)^{-1} \hat{x}_{k}^{s}=\left(\Sigma_{k}^{s}\right)^{-1} x_{k}+\left(\Sigma_{k}^{s}\right)^{-1 / 2} u_{k}^{s}
$$

with

$$
\left(\Sigma_{k}^{s}\right)^{-1} \hat{x}_{k}^{s}=\left(\Sigma_{k \mid k}^{f}\right)^{-1} \hat{x}_{k \mid k}^{f}+\left(\Sigma_{k \mid k+1}^{b}\right)^{-1} \hat{x}_{k \mid k+1}^{b},
$$

which constitute the usual two-filter smoothing formula.

\section{Double-sweep Smoother}

To derive the double sweep or Rauch-Tung-Striebel (RTS) smoother [20], it is convenient to introduce the conditional observation

$$
\omega_{k}\left(x_{k+1}\right)=X_{x_{k}, x_{k+1}}\{O\} \mid x_{k+1}
$$

representing the information about $x_{k}$ contained in all observations, conditioned on the knowledge of $x_{k+1}$. Taking into account the decomposition

$$
O=P_{k} \wedge o_{k+1} \wedge F_{k+2}
$$

and noting that, given $x_{k+1}, F_{k+2}$ does not contribute any information about $x_{k}$ ( $x_{k}$ does not appear in this observation), we get

$$
\omega_{k}\left(x_{k+1}\right)=\left(\hat{o}_{k \mid k}^{f} \wedge o_{k+1}\right) \mid x_{k+1} .
$$

This expression represents the forward sweep of the RTS smoother. It can be implemented together with the forward Kalman recursion (4.7a).

To get the smoothed observation $\hat{o}_{k}^{s}$, we take into account the decomposition

$$
R_{\circ} X_{x_{k}, x_{k+1}}\{O\}=M_{x_{k+1}}\left\{\omega_{k}\left(x_{k+1}\right)\right\} \wedge \hat{o}_{k+1}^{s}
$$

with $\omega_{k}\left(x_{k+1}\right) \perp \hat{o}_{k+1}^{s}$, which in view of the definition (4.26) of a smoothed observation, implies

$$
\hat{o}_{k}^{s}=R_{\circ} X_{x_{k}}\left\{M_{x_{k+1}}\left\{\omega_{k}\left(x_{k+1}\right)\right\} \wedge \hat{o}_{k+1}^{s}\right\} .
$$

This recursion constitutes the backward sweep of the RTS smoother.

Interpretation: To gain a better understanding of the RTS recursions (4.35), (4.37), we derive below a semi-explicit form of the smoother which relies on finding closed-form implementations of the primitives appearing in the recursions. However closed-form realizations 
of the primitives have the tendency to be less reliable numerically than the implicit implementations presented earlier. Thus, the explicit form of the RTS smoother presented below is for illustrative purposes only.

(i) Forward sweep: Assume again that $x_{k}$ is estimable from $P_{k}$. In addition, we assume that the noises $B_{k} u_{k}$ and $D_{k} u_{k}$ appearing in the dynamics $d_{k+1}$ and measurements $m_{k+1}$ are uncorrelated, i.e. $B_{k} D_{k}^{T}=0$. In this case, given $x_{k+1}, m_{k+1}$ does not contribute any information about $x_{k}$, so that

$$
\omega_{k}\left(x_{k+1}\right)=\left(\hat{o}_{k \mid k}^{f} \wedge d_{k+1}\right) \mid x_{k+1}
$$

where $\hat{o}_{k \mid k}^{f} \wedge d_{k+1}$ is given by (4.12). Premultiplying (4.12) by

$$
T_{k}=\left[\begin{array}{cc}
I & 0 \\
-A_{k} & I
\end{array}\right]
$$

and applying an orthonormal transformation $V_{k}$ to the vector $\left[v_{k}^{T} u_{k}^{T}\right]^{T}$, where $V_{k}$ is selected such that

$$
\left[\begin{array}{cc}
\Sigma_{k \mid k}^{f 1 / 2} & 0 \\
-A_{k} \Sigma_{k \mid k}^{f 1 / 2} & B_{k}
\end{array}\right] V_{k}^{T}=\left[\begin{array}{cc}
G_{k} & H_{k} \\
0 & J_{k}
\end{array}\right],
$$

the conditional observation $\omega_{k}\left(x_{k+1}\right)$ can be expressed as

$$
\omega_{k}\left(x_{k+1}\right): \quad \Theta_{k} \hat{x}_{k \mid k}^{f}+R_{k} x_{k+1}=x_{k}+G_{k} n_{k}
$$

with

$$
\Theta_{k}=I+H_{k} J_{k}^{-1} A_{k} \quad R_{k}=-H_{k} J_{k}^{-1} E_{k+1},
$$

where $n_{k} \sim \mathcal{N}(0, I)$. To interpret the matrices $\Theta_{k}, R_{k}$, and $G_{k}$ appearing in (4.41), note that by multiplying (4.40) by its transpose, we find

$$
\begin{aligned}
J_{k} J_{k}^{T} & =A_{k} \Sigma_{k \mid k}^{f} A_{k}^{T}+B_{k} B_{k}^{T} \triangleq \Sigma_{k+1 \mid k}^{f} \\
H_{k} J_{k}^{T} & =-\Sigma_{k \mid k}^{f} A_{k}^{T} \\
G_{k} G_{k}^{T} & =\Sigma_{k \mid k}^{f}-\Sigma_{k \mid k}^{f} A_{k}^{T}\left(\Sigma_{k+1 \mid k}^{f}\right)^{-1} A_{k} \Sigma_{k \mid k}^{f} \triangleq \Sigma_{k}^{p} .
\end{aligned}
$$

In the above expressions, we have assumed that the matrix $J_{k}$ is invertible. But, according to (4.43a), $J_{k}$ is a square-root of the covariance matrix $\Sigma_{k+1 \mid k}^{f}$ of the noise $B_{k} u_{k}-A_{k} \Sigma_{k \mid k}^{f 1 / 2} v_{k}$ in the predicted observation $\hat{o}_{k+1 \mid k}^{f}$. Thus, $J_{k}$ will be invertible whenever $\Sigma_{k+1 \mid k}^{f}$ is positive definite. Substituting (4.43a) inside the expressions (4.42) for $\Theta_{k}$ and $R_{k}$ gives

$$
\begin{aligned}
& \Theta_{k}=I-\Sigma_{k \mid k}^{f} A_{k}^{T}\left(\Sigma_{k+1 \mid k}^{f}\right)^{-1} A_{k} \\
& R_{k}=\Sigma_{k \mid k}^{f} A_{k}^{T}\left(\Sigma_{k+1 \mid k}^{f}\right)^{-1} E_{k+1} .
\end{aligned}
$$


Finally, from (4.41), we see that $G_{k} G_{k}^{T}=\Sigma_{k}^{p}$ is the filtered error variance for $x_{k}$ based on the observation $\omega_{k}\left(x_{k+1}\right)$, i.e based on the past observations, and the "pinned" value of $x_{k+1}$.

Backward sweep: Since the mutation of $x_{k+1}$ inside $\omega_{k}\left(x_{k+1}\right)$ is accomplished by moving the $R_{k} x_{k+1}$ term to the right hand side, the observation $M_{x_{k+1}}\left\{\omega_{k}\left(x_{k+1}\right)\right\} \wedge \hat{o}_{k+1}^{s}$ takes the form

$$
\left[\begin{array}{c}
\Theta_{k} \hat{x}_{k \mid k}^{f} \\
\hat{x}_{k}^{s \mid k}
\end{array}\right]=\left[\begin{array}{cc}
I & -R_{k} \\
0 & I
\end{array}\right]\left[\begin{array}{c}
x_{k} \\
x_{k+1}
\end{array}\right]+\left[\begin{array}{cc}
G_{k} & 0 \\
0 & \Sigma_{k+1}^{s 1 / 2}
\end{array}\right]\left[\begin{array}{c}
n_{k} \\
u_{k+1}^{s}
\end{array}\right] .
$$

To extract $x_{k}$ from this observation, we need only to premultiply it by [ $I R_{k}$ ], which gives (4.31) with

$$
\begin{aligned}
\hat{x}_{k}^{s} & =\Theta_{k} \hat{x}_{k \mid k}^{f}+R_{k} \hat{x}_{k+1}^{s} \\
\Sigma_{k}^{s} & =R_{k} \Sigma_{k+1}^{s} R_{k}^{T}+\Sigma_{k}^{p} .
\end{aligned}
$$

\section{Application to Failure Detection}

The descriptor Kalman filtering recursions of Section 4.A are directly applicable to a recently proposed [21] failure detection procedure for dynamical systems containing nuisance parameters, i.e., quantities taking arbitary unknown values. Given a standard state-space model where a nuisance term appears additively in the dynamics, the nuisance can be removed by premultiplying the dynamics with a matrix which nulls out the nuisance space. This yields a system with descriptor dynamics, where the presence or absence of failures can be determined by applying either innovations-based statistical failure detection tests, or algebraic tests relying on parity checks. Since the Kalman filtering recursions (4.7a)-(4.7c) generate both innovations and parity checks, they provide all the data necessary to implement failure detection schemes.

Our objective at this stage is not to discuss failure detection issues in detail, but just to illustrate the applicability of descriptor Kalman filtering algorithms. Accordingly, we follow the formulation of reference [21], to which the reader is referred for more extensive discussions and a comprehensive bibliography.

As starting point, we formulate the standard binary hypothesis testing problem for linear observation models in terms of the primitives introduced earlier. Given the measurement vector $y$, and two models

$$
\begin{aligned}
& \mathcal{H}_{0}: \quad E y=B u \\
& \mathcal{H}_{1}: \quad E y=A x+B u
\end{aligned}
$$

we seek to decide which one best fits $y$. Such a decision requires

(i) checking whether the constraints $\mathcal{S}\left\{\mathcal{H}_{0}\right\}$ and/or $\mathcal{S}\left\{\mathcal{H}_{1}\right\}$ are satisfied, and, if both are satisfied, then 
(ii) computing the $\log$-likelihood ratio $\log L\left\{\mathcal{H}_{1}\right\}-\log L\left\{\mathcal{H}_{0}\right\}$ for comparison to a selected threshold.

Performing this decision reduces therefore to computing

$$
\bar{R}\left\{\mathcal{H}_{0}\right\} \quad \text { and } \quad \bar{R}\left\{\mathcal{H}_{1}\right\} .
$$

A careful inspection of the canonical decomposition algorithm following Lemma 2.1 reveals that the numerical procedure required to compute $\bar{R}\left\{\mathcal{H}_{1}\right\}$ yields $\bar{R}\left\{\mathcal{H}_{0}\right\}$ at the same time.

Next, suppose we consider the same problem as before, but with an unknown nuisance vector $n$, so that we need to decide between $\mathcal{H}_{0}$ and $\mathcal{H}_{1}$, where

$$
\begin{aligned}
& \mathcal{H}_{0}: \quad E y=G n+B u \\
& \mathcal{H}_{1}: \quad E y=A x+G n+B u .
\end{aligned}
$$

In this case, all we need to do is first to extract $x$ from the observations $\mathcal{H}_{i}$ with $i=0,1$, which has the effect of removing the nuisance term $G n$, and then apply the previous hypothesis testing procedure to the extracted observations $X_{x}\left\{\mathcal{H}_{i}\right\}$. Again, because $X_{x}$ requires only the computation of the left null space of $G$, a single algorithm can be used to generate $X_{x}\left\{\mathcal{H}_{i}\right\}$ for $i=0,1$.

Finally, let us examine the failure detection problem with isolation studied in [21]. We are given the system

$$
\begin{aligned}
x_{k+1} & =A x_{k}+B v_{k}+G_{x} n_{k}+\Gamma u_{k}+f m_{k} \\
y_{k} & =C x_{k}+D v_{k}+G_{y} n_{k}
\end{aligned}
$$

where $x_{k}$ is the state of the system, $u_{k}$ the known input, $y_{k}$ the observed output, and $v_{k}$ a white Gaussian noise sequence such that $v_{k} \sim \mathcal{N}(0, I)$. The vector

$$
\left[\begin{array}{l}
G_{x} \\
G_{y}
\end{array}\right] n_{k}
$$

represents a nuisance modelling unobserved input and output disturbances or possible alternative failures not considered here. The term $f m_{k}$ represents the actuator failure that we seek to detect and isolate. For simplicity, it is assumed that the matrices apearing in the above dynamics are constant, and have appropriate dimensions. In the above model, the absence of failure corresponds to the case where $f=0$, whereas in the presence of a failure, the known vector $f \neq 0$. To cast the above model within our framework, we rewrite (4.50a)-(4.50b) as the observation

$$
\mathcal{H}_{k}^{i}:\left[\begin{array}{c}
-\Gamma u_{k} \\
y_{k}
\end{array}\right]=\left[\begin{array}{cccc}
-I & A & G_{x} & f \\
0 & C & G_{y} & 0
\end{array}\right]\left[\begin{array}{c}
x_{k+1} \\
x_{k} \\
n_{k} \\
m_{k}
\end{array}\right]+\left[\begin{array}{c}
B \\
D
\end{array}\right] v_{k} .
$$


The indices $k$ and $i$ appearing in $\mathcal{H}_{k}^{i}$ correspond respectively to the time index and hypothesis. Thus, $i=0$ in the absence of failure, and $i=1$ when a failure is present. The onset of a failure corresponds to $i$ jumping from 0 to 1 at a certain time $s$. We seek to detect such a jump, and estimate the failure time $s$. This can be accomplished by applying the following algorithm.

1) For $i=0,1$ and $k \geq 0$, compute

$$
X_{\left(x_{k+1}, x_{k}, m_{k}\right)}\left\{\mathcal{H}_{k}^{i}\right\} .
$$

Because the matrices $G_{x}$ and $G_{y}$ do not depend on either the choice of hypothesis or the time index $k$, the above extraction needs to be performed only once for $i=0,1$, and all $k \geq 0$; see the previous comment concerning the binary hypothesis problem with nuisance rejection.

2) Apply the Kalman filtering recursions (4.7a)-(4.7c), (4.8) to the observation stream

$$
O(s, k)=\left(\bigwedge_{l=0}^{s} X_{x_{l+1}, x_{l}, m_{l}}\left\{\mathcal{H}_{l}^{0}\right\}\right) \wedge\left(\bigwedge_{l=s+1}^{k} X_{x_{l+1}, x_{l}, m_{l}}\left\{\mathcal{H}_{l}^{1}\right\}\right)
$$

which models a situation where a failure has occured at time $s \leq k$ (the case $s=k$ corresponds to the absence of failure). Then, verify whether the measurement vectors belong to the constraint space $\mathcal{S}\{O(s, k)\}$, and compute

$$
\frac{\max _{k-L<s \leq k} L(O(s, k))}{L(O(k, k))} \quad \text { and } \quad \hat{s}=\arg \max _{k-L<s \leq k} L(O(s, k))
$$

for a properly chosen window length $L$. When the likelihood ratio exceeds a properly selected threshold, a failure occuring at time $\hat{s}$ has been detected.

Note that if the system under consideration is an interconnection of local subsystems, and the nuisance affects only a subsystem, the nuisance rejection of step 1) can be performed locally. However, the Kalman filtering phase of the algorithm remains global.

\section{$5 \quad$ Finite State Processes}

The previous results can be extended to the nonlinear nonGaussian case, when the parameter vector $X$ to be estimated belongs to a finite set $\mathcal{X}$.

\section{A Observation Model}

An observation $o$ consists as before of a pair $(\mathcal{C}, \mathbf{P})$ formed by a set $\mathcal{C}$ defining the allowable pairs of measurements $Y$ and parameter vector $X$, and, depending on whether the measurement vector $Y$ takes discrete or continuous values, a probability distribution

$$
\mathbf{P}(Y=y, X=x)=\mathbf{p}(y, x),
$$


or a density

$$
\mathbf{P}(Y \in d \mathcal{S}, X=x)=\mathbf{p}(y, x) d V
$$

with $(y, x) \in \mathcal{C}$, where $d V=|d \mathcal{S}|$ represents an element of volume of the set $\mathcal{S}$ of measurements $Y$ compatible with the constraint set $\mathcal{C}$. If $\mathcal{Y}$ and $\Pi_{\mathcal{Y}}$ denote respectively the domain of $y$, and the projection operator which associates to a pair $(y, x)$ its coordinate $y$, i.e.

$$
\Pi_{\mathcal{Y}}(y, x)=y,
$$

the set $\mathcal{S}$ of valid outputs is given by

$$
\mathcal{S}=\Pi_{\mathcal{Y}} \mathcal{C} .
$$

The reference measure $d V$ which is defined on $\mathcal{S}$ is left unspecified, since it depends on the set $\mathcal{S}$. Finally, given $y \in \mathcal{S}$, the set $\mathcal{X}(y)$ represents the set of parameter vectors $x$ such that the pair $(y, x) \in \mathcal{C}$.

Since $X$ belongs to a finite set, we shall focus our interest exclusively on ML estimates. As before, we say that $X$ is estimable if $\mathbf{p}(y, x)$ admits a unique maximum over $\mathcal{X}(y)$, in which case the ML estimate is defined as

$$
\hat{x}_{M L}(y)=\arg \max _{x \in \mathcal{X}(y)} \mathbf{p}(y, x) .
$$

The corresponding likelihood and conditional likelihood functions are then given by

$$
\begin{aligned}
\mathbf{q}(y) & =\mathbf{p}\left(y, \hat{x}_{M L}(y)\right)=\max _{x \in \mathcal{X}(y)} \mathbf{p}(y, x) \\
\mathbf{q}(x \mid y) & =\frac{\mathbf{p}(y, x)}{\mathbf{q}(y)}
\end{aligned}
$$

which are defined respectively over $\mathcal{S}$ and $\mathcal{X}(y)$. Thus, as in the Gaussian case, we have decomposed the pair $(\mathcal{C}, \mathbf{p}(y, x))$ into two subpairs $(\mathcal{S}, \mathbf{q}(y))$ and $(\mathcal{X}(y), \mathbf{q}(x \mid y))$ describing the marginal and conditional constraint sets and likelihood functions. Again, $\mathbf{q}(y)$ will be denoted as $L(o)$ to indicate its association with $o$.

However, unlike the Gaussian case, the likelihood and conditional likelihood functions $\mathbf{q}(y)$ and $\mathbf{q}(x \mid y)$ do not coincide with the marginal and conditional densities

$$
\begin{aligned}
\mathbf{p}(y) & =\sum_{x \in \mathcal{X}(y)} \mathbf{p}(y, x) \\
\mathbf{p}(x \mid y) & =\frac{\mathbf{p}(y, x)}{\mathbf{p}(y)}
\end{aligned}
$$

which differ from (5.6) and (5.7) by the fact that we are summing instead of maximizing over $x$. 


\section{B Primitives}

The primitives introduced in Section 3 for linear Gaussian observations can be generalized as follows. First, consider two observations $o_{i}=\left(\mathcal{C}_{i}, \mathbf{P}_{i}\right)$ with $i=1,2$, where the parameter vectors of $o_{1}$ and $o_{2}$ can be decomposed into attributes $x_{1}$ and $x_{2}$ which are specific to these observations, as well as a component $x_{c}$ common to both. Similarly the measurements vectors of $o_{1}$ and $o_{2}$ contain parts $y_{1}$ and $y_{2}$ which are specific to these observations, as well as a common part $y_{c}$. Then, adopting the notation (3.3a)-(3.3c) for the measurement and parameter vectors of each observation and their combination, the combined observation $o_{1} \wedge o_{2}$ is formed by the pair $(\mathcal{C}, \mathbf{P})$ such that

$$
\begin{aligned}
\mathcal{C} & =\mathcal{C}_{1} \wedge \mathcal{C}_{2} \\
& =\left\{(y, x):\left(\eta_{1}, \chi_{1}\right) \in \mathcal{C}_{1} \text { and }\left(\eta_{2}, \chi_{2}\right) \in \mathcal{C}_{2}\right\} \\
\mathbf{p}(y, x) & =\mathbf{p}_{1}\left(\eta_{1}, \chi_{1}\right) \mathbf{p}_{2}\left(\eta_{2}, \chi_{2}\right) .
\end{aligned}
$$

Because the probability distributions/densities we are considering are nonGaussian with finite valued parameter vectors, both $\mathbf{p}(y, x)$ and $\mathbf{q}(x \mid y)$ are minimal sufficient statistics for estimating $X$, i.e. we cannot base the reduction operation $R\{$.$\} on the need to extract a$ minimal sufficient observation from $o$. However, as indicated by (3.14), an important property of the reduction operation $R\{$.$\} in the Gaussian case is that it retains no information about$ the output $y$, in the sense that its likelihood function

$$
L(R\{o\})=1
$$

is uniformly distributed. The conditional likelihood function $\mathbf{q}(x \mid y)$ has precisely this characteristic, i.e.

$$
\max _{x} \mathbf{q}(x \mid y)=1 \text {. }
$$

This implies that the primitives $R\{$.$\} and \bar{R}\{$.$\} need to be defined as:$

$$
\begin{aligned}
& R\{o\}=\left(\mathcal{C}_{R}, \mathbf{q}(x \mid y)\right) \\
& \bar{R}\{o\}=(\mathcal{S}, \mathbf{q}(y)),
\end{aligned}
$$

where the set $\mathcal{C}_{R}$ is obtained by removing from $\mathcal{C}$ the constraints affecting $y$ alone, so that

$$
\mathcal{C}=\mathcal{C}_{R} \wedge \mathcal{S}
$$

Note that because the conditional likelihood function $\mathbf{q}(x \mid y)$ given by (5.7) is defined only on $\mathcal{C}$, the definition of $R\{o\}$ in (5.14) requires extending $\mathbf{q}(x \mid y)$ from $\mathcal{C}$ to the larger set $\mathcal{C}_{R}$, where the choice of extension does not matter, since only measurement vectors $y$ in $\mathcal{S}$ (and thus pairs $(x, y)$ in $\mathcal{C}$ ) will ultimately be allowed.

The mutation corresponds again to the change of status of a parameter or measurement from unknown to known, or vice versa, and the conditional observation and extraction macros are defined by (3.28a)-(3.28b), so that for an observation $o$ with density $\mathbf{p}\left(y, x_{0}, x_{1}\right)$, the density of the extracted observation $X_{x_{1}}\{0\}$ is given by

$$
\mathbf{q}\left(y, x_{1}\right)=\max _{x_{0}} \mathbf{p}\left(y, x_{0}, x_{1}\right) \text {. }
$$

$\mathrm{RR} \mathrm{n}^{\circ} 2088$ 


\section{Estimation of Hidden Markov Models}

To illustrate the application of the above macros, we consider the problem of finding the most likely state sequence of a hidden Markov model (HMM). In such models, the states of a Markov chain are not observed directly, but need to be estimated from a sequence of measurements depending indirectly on the states. Models of this type are often used in speech processing [8], or in digital communications for decoding convolutional codes or the deconvolution of intersymbol interference [22], [23], [24].

Let $x_{k}$ be a Markov chain defined for $0 \leq k \leq N$, taking values in a finite set $\mathcal{X}$. The joint probability distribution of the chain can be expressed as

$$
\mathbf{p}(X)=\mathbf{q}_{i}\left(x_{0}\right) \mathbf{q}_{e}\left(x_{N}\right) \prod_{k=0}^{N-1} \tau\left(x_{k}, x_{k+1}\right) .
$$

This form is a slight variant of the standard expression for Markov processes, which is sometimes employed [25] to model Markov processes for which some information about both the initial and final state is available. This situation occurs for example when we use a convolutional encoder to transmit data blocks separated by zero bits whose role is to ensure that the encoder starts and terminates in a known state (the zero state). For such a case, we have

$$
\mathbf{q}_{i}(x)=\mathbf{q}_{e}(x)=\delta(x),
$$

where $\delta(x)$ denotes the distribution equal to one for $x=0$, and zero otherwise.

The observations $y_{k}$ with $0 \leq k \leq N-1$ may take either continuous or discrete values. Given a complete state trajectory, they are modeled by the conditional density/distribution

$$
\mathbf{p}(Y \mid X)=\prod_{k=0}^{N-1} \phi\left(y_{k}, x_{k}, x_{k+1}\right),
$$

whose form indicates that $y_{k}$ may be either a measurement of the state $x_{k}$ or of the transition from $x_{k}$ to $x_{k+1}$. For simplicity, we assume that there are no constraints on the states $x_{k}$ and observations $y_{k}$, so that an observation $o$ will consist only of a probability distribution/density.

Then the joint distribution/density for the measurements and states can be expressed as

$$
\mathbf{p}(Y, X)=\mathbf{p}(Y \mid X) \mathbf{p}(X)=\bigwedge_{j=0}^{N+1} o_{j}
$$

where

$$
o_{k+1}: \quad \tau\left(x_{k}, x_{k+1}\right) \phi\left(y_{k}, x_{k}, x_{k+1}\right)
$$

for $0 \leq k \leq N-1$, and

$$
o_{0}: \mathbf{q}_{i}\left(x_{0}\right) \quad o_{N+1}: \quad \mathbf{q}_{e}\left(x_{N}\right) .
$$




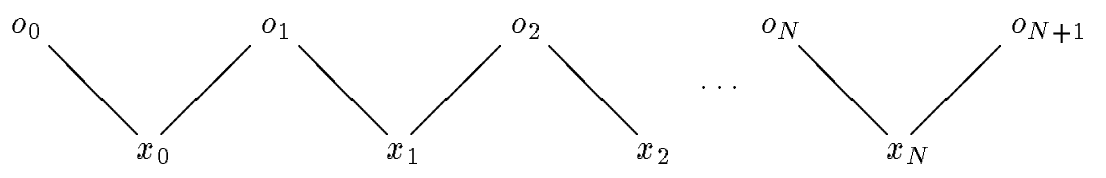

Figure 1: Linear XO-graph.

The maximum likelihood sequence estimation problem for HMMs consists in jointly maximizing $\mathbf{p}(Y, X)$ with respect to the variables $x_{k}$ with $0 \leq k \leq N$. The best known solution of this problem is the celebrated Viterbi algorithm [26], [22]. To relate this algorithm to those discussed in Section 4 for descriptor systems, it is useful to construct the XO-graph associated to the observations (5.22)-(5.23). The concept of XO-graph was introduced in [7] for linear Gaussian observations, but it applies also to the nonGaussian case. Its role is to display the dependency structure of the observations with respect to the variables to be estimated. Conceptually, it is similar to the graph representing the neighborhood structure of a Markov random field [9]. In such a graph, the observations $o_{j}$ and variables $x_{k}$ are represented respectively by $o$ - and $x$-nodes. An edge connects the nodes $o_{j}$ and $x_{k}$ if the variable $x_{k}$ appears in the probability distribution/density corresponding to $o_{j}$. For the HMM considered here, $o_{k+1}$ is connected only to the nodes $x_{k}$ and $x_{k+1}$, so that the HMM (5.22)-(5.23) admits the linear graph structure shown in Fig. 1.

But the descriptor estimation problem of Section 4 admits exactly the same XO-graph! Since the structure of this graph determines completely the order in which observations are processed and the variables $x_{k}$ are estimated, we can immediately conclude that the filtering and smoothing algorithms described in Section 4 are directly applicable to the HMM considered here. However, since the primitives are not implemented in the same manner, it is of interest to examine more precisely the internal mechanics and outcome of each algorithm.

Forward pass: Let $P_{k}$ be the combination (4.5) of all observations in the past of time $k$. Then, the filtered observation $\hat{o}_{k \mid k}^{f}$ given by (4.6a) corresponds actually to the function $\mathbf{q}_{k}^{f}\left(x_{k}\right)$ measuring the likelihood of the best path terminating in state $x_{k}$ given the measurements $y_{1}, \ldots, y_{k-1}$. Because the definition of $\hat{o}_{k \mid k}^{f}$ includes a reduction, the distribution $\mathbf{q}_{k}^{f}\left(x_{k}\right)$ is normalized, i.e. its maximum value over all $x_{k}$ 's is one. A similar normalization is often performed in convolutional decoders by subtracting periodically a constant from the value function $J_{k}^{f}\left(x_{k}\right)=-\ln \mathbf{q}_{k}^{f}\left(x_{k}\right)$ to prevent it from growing excessively. This is possible because we are only interested in the relative value of $J_{k}^{f}$ or $\mathbf{q}_{k}^{f}$ for different $x_{k}$ 's. Then, if we consider the recursion (4.7a), the extracted observation $X_{x_{k+1}}\left\{\hat{o}_{k \mid k}^{f} \wedge o_{k+1}\right\}$ is specified by the distribution

$$
\xi_{k+1}^{f}\left(x_{k+1}\right)=\max _{x_{k}} \mathbf{q}_{k}^{f}\left(x_{k}\right) \tau\left(x_{k}, x_{k+1}\right) \phi\left(y_{k}, x_{k}, x_{k+1}\right),
$$


where the argument of the maximum is given by

$$
x_{k}^{*}=\psi_{k+1}\left(x_{k+1}\right),
$$

and the dependence of $x_{k}^{*}$ on the known observation $y_{k}$ has been suppressed. Given that the state of the Markov chain at time $k+1$ is $x_{k+1}$ and the observations up to time $k$ are $y_{0}$, $\ldots y_{k}, x_{k}^{*}=\psi_{k+1}\left(x_{k+1}\right)$ represents the most likely state at time $k$. The function $\psi_{k+1}($.$) is$ usually called the back-pointer function, since given the current state at time $k+1$, it points to the most likely state at the previous instant.

Then, if

$$
\begin{aligned}
\lambda_{k+1} & =\bar{R}_{\mathrm{o}} X_{x_{k+1}}\left\{\hat{o}_{k \mid k}^{f} \wedge o_{k+1}\right\} \\
& =\max _{x_{k+1}} \xi_{k+1}^{f}\left(x_{k+1}\right)
\end{aligned}
$$

we have

$$
\mathbf{q}_{k+1}^{f}\left(x_{k+1}\right)=\xi_{k+1}^{f}\left(x_{k+1}\right) / \lambda_{k+1} .
$$

Furthermore, according to $(4.7 \mathrm{c})$, if $L_{k}$ denotes the likelihood function of the observation $P_{k}$, it satisfies the recursion

$$
L_{k+1}=\lambda_{k+1} L_{k},
$$

which can be used to evaluate the likelihood function of the measurement sequence.

According to (4.8), the forward filter can be initialized with

$$
\begin{aligned}
L_{0} & =\lambda_{0}=\max _{x_{0}} \mathbf{q}_{i}\left(x_{0}\right) \\
\mathbf{q}_{0}^{f}\left(x_{0}\right) & =\mathbf{q}_{i}\left(x_{0}\right) / \lambda_{0} .
\end{aligned}
$$

Viterbi/double sweep decoder: If $O$ represents the combined observation (5.21), the smoothed observation $\hat{o}_{k}^{s}$ given by (4.26) is the distribution $\mathbf{q}_{k}^{s}\left(x_{k}\right)$ obtained after maximizing $\mathbf{p}(Y, X)$ with respect to all $x_{l}$ with $l \neq k$. Its maximum

$$
\hat{x}_{k}=\arg \max _{x_{k}} \mathbf{q}_{k}^{s}\left(x_{k}\right)
$$

represents the most likely state at time $k$, i.e. the most likely trajectory corresponding to the measurements $y_{k}$ with $0 \leq k \leq N-1$ goes through $\hat{x}_{k}$ at time $k$.

From the definition of the conditional observation macro, we find that the observation $\omega_{k}\left(x_{k+1}\right)=\hat{o}_{k \mid k}^{f} \wedge o_{k+1} \mid x_{k+1}$ admits the distribution

$$
\mathbf{q}_{k}^{f}\left(x_{k}\right) \tau\left(x_{k}, x_{k+1}\right) \phi\left(y_{k}, x_{k}, x_{k+1}\right) / \mathbf{q}_{k+1}^{f}\left(x_{k+1}\right)
$$

so that according to (4.36), the observation $R_{\circ} X_{x_{k}, x_{k+1}}\{O\}$ has for distribution

$$
\mathbf{q}_{k}^{f}\left(x_{k}\right) \tau\left(x_{k}, x_{k+1}\right) \phi\left(y_{k}, x_{k}, x_{k+1}\right) \mathbf{q}_{k+1}^{s}\left(x_{k+1}\right) / \mathbf{q}_{k+1}^{f}\left(x_{k+1}\right) .
$$


Its joint maximum over $x_{k}$ and $x_{k+1}$ gives the most likely states $\hat{x}_{k}$ and $\hat{x}_{k+1}$ at times $k$ and $k+1$. Maximizing over $x_{k}$ first, and noting that the $x_{k}$ dependent part of (5.31) is identical to the one minimized in $(5.24 \mathrm{a})$ yields

$$
\hat{x}_{k}=\psi_{k+1}\left(\hat{x}_{k+1}\right) .
$$

Thus, the most likely trajectory is obtained through a double sweep algorithm, where the forward sweep computes the trace back function $\psi_{k+1}($.$) , and the backward sweep generates$ the optimum state trajectory, starting from the most likely final state $\hat{x}_{N}$. This state can be computed by observing that the observation $\hat{o}_{N}^{s}=\hat{o}_{N \mid N}^{f} \wedge o_{N+1}$ has for distribution

$$
\mathbf{q}_{N}^{s}\left(x_{N}\right)=\mathbf{q}_{N}^{f}\left(x_{N}\right) \mathbf{q}_{e}\left(x_{N}\right),
$$

whose maximum yields $\hat{x}_{N}$.

By employing primitives which are sensitive to data type, i.e. which act differently on Gaussian and finite state processes, we have therefore been able to show that the Viterbi and Rauch-Tung-Striebel double-sweep algorithms are almost identical, as was first noted informally in [10]. Of course, one could push forward the analogy existing between the ML estimation problems for descriptor systems and HMMs by attempting to develop a "twofilter" decoder for the most-likely state sequence of a HMM. However, such a decoder would be less attractive than the Viterbi decoder, since it would require the solution of two dynamic programming problems, one for each time direction.

\section{Conclusions}

In this paper we have developed a set of high level primitives for ML estimation which, because they rely on general statistical concepts, are applicable to estimation problems where sufficient statistics admit finite parametrizations, such as linear Gaussian models or finite-state processes. The power of these high level primitives has been illustrated by considering the ML estimation problems for linear descriptor systems and hidden Markov models.

The work presented here can be extended in at least two directions. First, for the finitestate case, we have assumed that observations can be modeled by a distribution/density and constraint set pair $(\mathbf{p}(y, x), \mathcal{C})$. However for certain applications, such as queing networks, the sigma field of events that can be probabilized is more restricted, so that an observation needs to be modeled as a triple $(\Omega, \mathcal{A}, \mathbf{P})$ formed by a sample space, sigma field, and probability measure. In this case, because of the possibility that different observations might have common components, certain technical difficulties arise in the definition of the combinator $\wedge$, which are examined in [6].

Another area where the primitives developed in this paper are expected to be useful is for the formulation of stochastic dynamic programming problems involving either linear Gaussian systems or controlled Markov chains.

$\mathrm{RR} \mathrm{n}^{\circ} 2088$ 
A third direction of research concerns the large amount of flexibility existing in the choice of recursive strategies for solving ML estimation problems specified by coupled observations. For the smoothing problem of descriptor systems, the two-filter and double-sweep smoothers are smoothers are only two of several possible processing strategies. A third procedure consists for example of processing the observations outward from the center of the interval, until the edges are reached, and then performing a second inward pass. The choice of possible algorithms becomes even larger when a network of interconnected descriptor systems is considered, since in this case locality can be exploited both in time and in space. This raises therefore the question of finding a way to describe all processing strategies within a single framework, in order to be able to select optimum algorithms in terms of memory requirements and computational cost.

\section{Acknowledgements}

The authors would like to thank Dr. Michèle Basseville of IRISA and Prof. Alan Willsky of MIT for several valuable suggestions concerning both the contents and presentation of this paper.

\section{References}

[1] H. W. Sorenson, Kalman Filtering: Theory and Application. New York: IEEE Press, 1985.

[2] B. D. O. Anderson and J. B. Moore, Optimal Filtering. Englewood Cliffs, NJ: PrenticeHall, 1979.

[3] G. J. Bierman, Factorization Methods for Discrete Sequential Estimation. New York: Academic Press, 1977.

[4] T. Kailath, Lectures on Wiener and Kalman Filtering. Berlin: Springer Verlag, 1985.

[5] P. Le Guernic, T. Gauthier, M. Le Borgne, and C. Le Maire, "Programming real-time applications with SignaL" Proc. IEEE, vol. 79, pp. 1321-1336, Sep. 1991.

[6] A. Benveniste, "Constructive probability and the Signalea language: building and handling random processes with programming," Tech. Rep. 1532, Institut National de Recherche en Informatique et Automatique, Rocquencourt, France, Oct. 1991.

[7] R. Nikoukhah, D. Taylor, B. C. Levy, and A. S. Willsky, "Graph structure and recursive estimation of noisy linear relations," Tech. Rep. 1912, Institut National de Recherche en Informatique et Automatique, Rocquencourt, France, May 1993.

[8] L. R. Rabiner and B. H. Juang, "An introduction to hidden Markov models," IEEE ASSP Magazine, vol. 3, pp. 4-16, Jan. 1986. 
[9] R. Kindermann and J. L. Snell, Markov Random Fields and their Applications. Providence, RI: American Mathematical Society, 1980.

[10] B. Delyon, "Remarks on filtering of semi-Markov data," Tech. Rep. 733, Institut National de Recherche en Informatique et Systèmes Aléatoires, Rennes, France, May 1993.

[11] E. L. Lehmann, Theory of Point Estimation. Pacific Grove, CA: Wadsworth \& Brooks Cole, 1991.

[12] X. Wang and P. Bernhard, "Filtrage et lissage des systèmes discrets," Tech. Rep. 1083, Institut National de Recherche en Informatique et Automatique, Rocquencourt, France, Aug. 1989.

[13] R. Nikoukhah, A. S. Willsky, and B. C. Levy, "Kalman filtering and Riccati equations for descriptor sytems," IEEE Trans. Automat. Contr., vol. 37, pp. 1325-1342, Sep. 1992.

[14] L. Chisci and G. Zappa, "Square-root Kalman filtering of descriptor systems," Systems Control Lett., vol. 19, pp. 325-334, Oct. 1992.

[15] D. Taylor and A. S. Willsky, "Maximum likelihood estimation for two-point boundaryvalue descriptor systems," in Proc. 1991 Conf. Information Sciences and Systems, March 1991.

[16] A. S. Willsky, "A survey of design methods for failure detection in dynamic systems," Automatica, vol. 12, pp. 601-611, 1976.

[17] E. Y. Chow and A. S. Willsky, "Analytical redundancy and the design of robust failure detection systems," IEEE Trans. Automat. Contr., vol. 29, pp. 603-614, July 1984.

[18] D. Q. Mayne, "A solution of the smoothing problem for linear dynamic systems," Automatica, vol. 4, pp. 73-92, Nov. 1966.

[19] D. C. Fraser, A New Technique for the Optimal Smoothing of Data. PhD thesis, Dept. Aeronautics and Astronautics, M.I.T., Cambridge, MA, Jan. 1967.

[20] H. E. Rauch, F. Tung, and C. T. Striebel, "Maximum likelihood estimates of linear systems," AIAA Journal, vol. 3, pp. 1445-1450, Aug. 1965.

[21] A. Benveniste, M. Basseville, R. Nikoukhah, and A. S. Willsky, "On the use of descriptor systems for failure detection and isolation," in Proc. IFAC World Congress, (Sidney, Australia), pp. 497-500, July 1993.

[22] G. D. Forney, "The Viterbi algorithm," Proc. IEEE, vol. 61, pp. 268-278, March 1973.

[23] G. D. Forney, "Maximum-likelihood sequence estimation of digital sequences in the presence of intersymbol interferences," IEEE Trans. Inform. Theory, vol. 18, pp. 363378, May 1972.

$\mathrm{RR} \mathrm{n}^{\circ} 2088$ 
[24] A. J. Viterbi and J. K. Omura, Principles of Digital Communication and Coding. New York: McGraw-Hill, 1979.

[25] B. Jamison, "Reciprocal processes," Z. Wahrscheinlichkeitstheorie verw. Gebiete, vol. 30, pp. 65-86, 1974.

[26] A. J. Viterbi, "Error bounds for convolutional codes and an asymptotic optimum decoding algorithm," IEEE Trans. Inform. Theory, vol. 13, pp. 260-269, Apr. 1967. 
Unité de recherche INRIA Lorraine, Technopôle de Nancy-Brabois, Campus scientifique, 615 rue du Jardin Botanique, BP 101, 54600 VILLERS LĖS NANCY

Unité de recherche INRIA Rennes, Irisa, Campus universitaire de Beaulieu, 35042 RENNES Cedex Unité de recherche INRIA Rhône-Alpes, 46 avenue Félix Viallet, 38031 GRENOBLE Cedex 1

Unité de recherche INRIA Rocquencourt, Domaine de Voluceau, Rocquencourt, BP 105, 78153 LE CHESNAY Cedex

Unité de recherche INRIA Sophia-Antipolis, 2004 route des Lucioles, BP 93, 06902 SOPHIA-ANTIPOLIS Cedex

Éditeur

INRIA, Domaine de Voluceau, Rocquencourt, BP 105, 78153 LE CHESNAY Cedex (France)

ISSN 0249-6399 